\title{
High-order central Hermite WENO schemes: dimension-by-dimension moment-based reconstructions*
}

\author{
April 8, 2016 \\ Zhanjing Tao ${ }^{a}$, Fengyan $\mathrm{Li}^{b}$ and Jianxian Qiu ${ }^{a, 1}$ \\ ${ }^{a}$ School of Mathematical Sciences and Fujian Provincial Key Laboratory of Mathematical Modeling and \\ High-Performance Scientific Computing, Xiamen University, Xiamen, Fujian 361005, P.R. China. \\ ${ }^{b}$ Department of Mathematical Science, Rensselaer Polytechnic Institute, 110 8th Street, Troy NY 12180, United \\ States.
}

\begin{abstract}
In this paper, a class of high-order central finite volume schemes is proposed for solving one- and two-dimensional hyperbolic conservation laws. Formulated on staggered meshes, the methods involve Hermite WENO (HWENO) spatial reconstructions, and Lax-Wendroff type discretizations or the natural continuous extension of Runge-Kutta methods in time. Different from the central Hermite WENO methods we developed previously in [J. Comput. Phys. 281:148-176, 2015], the spatial reconstructions, a core ingredient of the methods, are based on the zeroth-order and the first-order moments of the solution, and are implemented through a dimension-by-dimension strategy when the spatial dimension is higher than one. This leads to much simpler implementation of the methods in higher dimension and better cost efficiency. Meanwhile, the proposed methods have the attractive features of the general central Hermite WENO methods such as being compact in reconstruction and requiring neither flux splitting nor numerical fluxes, while being accurate and essentially non-oscillatory. A collection of oneand two- dimensional numerical examples is presented to demonstrate high resolution and robustness of the methods in capturing smooth and non-smooth solutions.
\end{abstract}

Keywords: Finite volume method; central scheme; Hermite WENO; Lax-Wendroff; natural continuous extension (NCE) of Runge-Kutta.

\footnotetext{
*The research was partially supported by NSFC grant 11571290 and NSF grant DMS-1318409.

${ }^{1}$ Corresponding author. tzjnchy555@163.com (Z. Tao), lif@rpi.edu (F. Li), jxqiu@xmu.edu.cn (J. Qiu).
} 


\section{Introduction}

This paper is concerned with designing accurate and robust numerical methods for simulating hyperbolic conservation laws, namely

$$
\left\{\begin{array}{l}
u_{t}+\nabla \cdot f(u)=0 \\
u(x, 0)=u_{0}(x)
\end{array}\right.
$$

with suitable initial and boundary conditions. Here (1.1) can be scalar or a system, and it is often nonlinear. Hyperbolic conservation laws arise in a wide range of applications in science and engineering, such as aerodynamics, meteorology and weather prediction, astrophysical modeling, multi-phase flow problems, and the study of explosion and blast waves. In general the exact solutions of the nonlinear equations are not available, and discontinuity may appear in the solutions of such equations regardless of the smoothness of the initial and boundary data. Various numerical methods have been developed for solving hyperbolic conservation laws, such as finite difference methods [3,15,27], finite volume methods [9,21,29,30], finite element type methods (including streamline diffusion methods $[13,14]$ and discontinuous Galerkin methods [5-7]), and spectral methods $[10,33]$ etc.

WENO (weighted essentially non-oscillatory) methods within finite difference or finite volume frameworks have made great success in simulating hyperbolic conservation laws with their excellent balance between accuracy and non-oscillatory property. The first WENO scheme was constructed by Liu, Osher and Chan [21] in 1994 as a third-order finite volume method for one space dimension. Ever since then, the methods have been further developed for higher dimensions and with better accuracy, and also regarding general strategies for smoothness indicators and nonlinear weights, as well as the techniques to handle negative linear weights etc $[3,9,12,15,19,26,30,31]$. To improve its compactness while achieving high order accuracy, Hermite WENO (HWENO) methods are further developed [28, 29,39]. Here not only the solution but also its first spatial derivative(s) are evolved in time, and they are used in the high-order spatial reconstructions. With the same stencil, HWENO reconstructions can achieve higher order accuracy than standard WENO reconstructions.

WENO reconstructions were originally used in upwind or Godunov methods setting. They were then integrated into the central framework $[19,20,26]$, to further gain the advantages of central schemes, such as being free of numerical fluxes, relatively better stability, and no need for applying flux splitting etc. Compared to upwind type schemes, central schemes are also a class of effective schemes for hyperbolic conservation laws and they are relatively simpler. A second-order central scheme was first developed by Nessyahu and Tadmor [25] in 1990. Motivated by its simplicity and robustness, various high-order or semi-discrete versions of central schemes as well as their extensions to multiple dimensions were explored in $[1,4,16,17,19,20,22,23,26]$. Though it was observed that the local characteristic decomposition is not necessary for lower order central schemes, it has been shown [26] that such decomposition is still needed to control spurious oscillations when the accuracy order of the methods is higher.

In [34], we proposed high-order central HWENO (C-HWENO) schemes for solving the hyperbolic conservation laws in one and two space dimensions. The methods use HWENO reconstructions as spatial discretizations, and Lax-Wendroff type discretizations or the natural continuous extension of Runge-Kutta methods as time discretizations, in a central finite volume formulation on staggered meshes. Like all WENOtype methods, the C-HWENO methods are accurate and essentially non-oscillatory. Compared with WENO schemes, one major advantage of HWENO schemes is the compactness in the spatial reconstruction. Compared with the original HWENO schemes, the C-HWENO methods require neither flux splitting nor the use of numerical fluxes that are often exact or approximate Riemann solvers. 
The work in this paper is a continuation of the development in [34], with the same focus on the design of C-HWENO methods on structured meshes for hyperbolic conservation laws. In [34], the HWENO reconstructions are based on the solution and its derivative(s), while in the present work, we propose new HWENO reconstructions which are based on the zeroth-order and the first-order moments of the solution. Using moments of the solution itself is not new in HWENO reconstruction, with examples including the limiting procedure for DG methods in [28,29], the hybrid RKDG-HWENO reconstruction in [2], and $P_{N} P_{M}$ methods in $[8,24]$. One novel aspect of our current strategy is to involve all the first-order moments, including the mixed-type moment (see $\overline{v w}_{i j}^{n}$ in Section 2.2), of the solution, when the spatial dimension is higher than one [38]. This is an analogue of the tenor-type or called $Q$-type finite elements in the setting of finite element methods. Working with such choice of the moments enables a dimension-by-dimension procedure for the reconstructions, a core ingredient of the proposed methods. The HWENO reconstructions proposed here for two dimensions essentially use several one-dimensional reconstructions. This will improve the computational efficiency, as well as the ease of implementation in higher dimensions. Meanwhile, the proposed methods have the similar advantages as the C-HWENO methods in [34]. Like in [34], we apply in time the Lax-Wendroff type discretizations $[11,18,27]$ and the natural continuous extension of Runge-Kutta methods $[4,19,20,26,37]$.

The organization of this paper is as follows. In Section 2, we describe in detail the construction and implementation of the proposed C-HWENO schemes based on moments of the solution with Lax-Wendroff type time discretizations for the problem (1.1) in one and two dimensions. The two-dimensional spatial reconstructions are implemented in a dimension-by-dimension fashion. In Section 3, we present the construction and implementation of the proposed C-HWENO schemes with the natural continuous extension of Runge-Kutta methods as time discretizations. The HWENO reconstructions in Section 2 and Section 3 are partially the same. In Section 4, extensive numerical examples are provided to demonstrate the performance of the proposed schemes for smooth and nonsmooth examples in one and two dimensions. Concluding remarks are made in Section 5.

\section{Central Hermite WENO schemes with Lax-Wendroff time dis- cretization}

In this section we describe in detail the construction and implementation of central Hermite WENO (HWENO) schemes with fifth-order spatial reconstructions and Lax-Wendroff time discretizations for oneand two-dimensional scalar and system of conservation laws.

\subsection{One-dimensional case}

Consider the one-dimensional scalar conservation law

$$
\left\{\begin{array}{l}
u_{t}+f(u)_{x}=0, \\
u(x, 0)=u_{0}(x) .
\end{array}\right.
$$

The proposed numerical method will be defined on staggered meshes. For simplicity of presentation, uniform meshes are used with the mesh size $\Delta x$. Each cell of the primal mesh is denoted as $I_{i}=\left[x_{i-1 / 2}, x_{i+1 / 2}\right]$ with its cell center $x_{i}=\frac{1}{2}\left(x_{i-1 / 2}+x_{i+1 / 2}\right)$; and each cell of the $d u a l$ mesh is denoted as $I_{i+1 / 2}=\left[x_{i}, x_{i+1}\right]$ with its cell center $x_{i+1 / 2}=\frac{1}{2}\left(x_{i}+x_{i+1}\right)$. It will be seen that the primal and the dual meshes will be used in a staggered fashion along the time direction in the proposed scheme. 
Next we will proceed to derive our proposed method. In addition to the governing equation (2.1), we will also utilize the following equation,

$$
u_{t} \frac{x-x_{c}}{\Delta x}+f(u)_{x} \frac{x-x_{c}}{\Delta x}=0,
$$

where $x_{c}$ is the center of the relevant mesh element. In particular, for a cell $I_{i}$ from the primal mesh, we will take $x_{c}=x_{i}$; for a cell $I_{i+1 / 2}$ from the dual mesh, we will take $x_{c}=x_{i+1 / 2}$. We now integrate (2.1) and (2.2) over $I_{i+1 / 2} \times\left[t^{n}, t^{n+1}\right]$, apply integration by parts, and get

$$
\begin{gathered}
\frac{1}{\Delta x} \int_{I_{i+1 / 2}} u\left(x, t^{n+1}\right) d x=\frac{1}{\Delta x} \int_{I_{i+1 / 2}} u\left(x, t^{n}\right) d x-\frac{1}{\Delta x} \int_{t^{n}}^{t^{n+1}}\left[f\left(u\left(x_{i+1}, t\right)\right)-f\left(u\left(x_{i}, t\right)\right)\right] d t, \\
\frac{1}{\Delta x} \int_{I_{i+1 / 2}} u\left(x, t^{n+1}\right) \frac{x-x_{i+1 / 2}}{\Delta x} d x=\frac{1}{\Delta x} \int_{I_{i+1 / 2}} u\left(x, t^{n}\right) \frac{x-x_{i+1 / 2}}{\Delta x} d x \\
-\frac{1}{\Delta x} \int_{t^{n}}^{t^{n+1}}\left[\frac{1}{2}\left[f\left(u\left(x_{i+1}, t\right)\right)+f\left(u\left(x_{i}, t\right)\right)\right]-\frac{1}{\Delta x} \int_{x_{i}}^{x_{i+1}} f(u(x, t)) d x\right] d t .
\end{gathered}
$$

Here $\Delta t=t^{n+1}-t^{n}$ is the time step.

Since our proposed method will be fifth-order accurate in space, we will approximate the line integral of $f(u)$ in $x$-variable from (2.4) with the four-point Gauss-Lobatto quadrature formula, namely,

$$
\frac{1}{\Delta x} \int_{x_{i}}^{x_{i+1}} f(u(x, t)) d x \approx \sum_{l=1}^{4} \omega_{l} f\left(u\left(\mathcal{G}_{l}^{x}, t\right)\right)
$$

where $\omega_{1}=\omega_{4}=\frac{1}{12}$ and $\omega_{2}=\omega_{3}=\frac{5}{12}$ are the quadrature weights and

$$
\mathcal{G}_{1}^{x}=x_{i}, \quad \mathcal{G}_{2}^{x}=x_{i+\frac{1}{2}-\frac{\sqrt{5}}{10}}, \quad \mathcal{G}_{3}^{x}=x_{i+\frac{1}{2}+\frac{\sqrt{5}}{10}}, \quad \mathcal{G}_{4}^{x}=x_{i+1},
$$

are the quadrature points over the dual cell $I_{i+1 / 2}$. Here and below, $x_{r}$ with non-integer $r$ should be understood as a natural linear interpolation of $\left\{x_{i}\right\}_{i}$ with respect to the subindex. This similarly goes to $y_{r}$. Associated with a cell $I_{i}$ of the primal mesh, we define

$$
\mathcal{G}^{x, i}=\left\{x_{i-\frac{1}{2}+\frac{\sqrt{5}}{10}}, x_{i}, x_{i+\frac{1}{2}-\frac{\sqrt{5}}{10}}\right\} .
$$

We also let $\mathcal{G}^{x}=\cup_{i} \mathcal{G}^{x, i}$.

Let's for the moment consider the problem (2.1) with $\left.u\right|_{I_{i}}$ as a constant at $t^{n}, \forall i$, for a reason which will become clear once the scheme is given. Under the assumption that the time step $\Delta t$ satisfies a CFL restriction $\Delta t \leq \frac{C_{c f l} \Delta x}{\max \left|f^{\prime}(u)\right|}$ with some constant $C_{c f l}$, the discontinuities starting at $t^{n}$ from $x_{i-\frac{1}{2}}$ and $x_{i+\frac{1}{2}}$ will not propagate to $x_{*}$ with $x_{*} \in \mathcal{G}^{x, i}$ over a single time step $\Delta t$, and therefore the solution of this problem restricted at $x_{*}$ with $x_{*} \in \mathcal{G}^{x, i}, \forall i$ are smooth for $t \in\left(t^{n}, t^{n+1}\right)$. Motivated by this, the flux function $f\left(u\left(x_{*}, t\right)\right)$ in $(2.3)$ and $(2.4)$ (with the last term replaced by $(2.5)$ ) will be approximated by a temporal Taylor expansion at $t^{n}$ as follows,

$$
f\left(u\left(x_{*}, t\right)\right) \approx f\left(u\left(x_{*}, t^{n}\right)\right)+\left(t-t^{n}\right) f_{t}\left(u\left(x_{*}, t^{n}\right)\right)+\frac{\left(t-t^{n}\right)^{2}}{2} f_{t t}\left(u\left(x_{*}, t^{n}\right)\right) .
$$

This corresponds to a third-order accuracy in time, and will be considered in the present work. In general, to achieve $(k+1)$ th-order accuracy in time, one would need to keep the first $k$ time derivatives on the right-hand side of (2.8). 
Using (2.8), the temporal integration of $f(u)$ can be approximated straightforwardly,

$$
\frac{1}{\Delta t} \int_{t^{n}}^{t^{n+1}} f\left(u\left(x_{*}, t\right)\right) d t \approx F\left(u\left(x_{*}, t^{n}\right)\right),
$$

where

$$
F(u)=f(u)+\frac{\Delta t}{2} f_{t}(u)+\frac{\Delta t^{2}}{6} f_{t t}(u)=f(u)+\frac{\Delta t}{2} f^{\prime}(u) u_{t}+\frac{\Delta t^{2}}{6}\left(f^{\prime \prime}(u)\left(u_{t}\right)^{2}+f^{\prime}(u) u_{t t}\right) .
$$

Following the idea of the Lax-Wendroff time discretization, the temporal derivative terms in (2.10) are further converted into spatial ones by repeatedly using the governing equation (2.1),

$$
\begin{aligned}
& u_{t}=-f(u)_{x}=-f^{\prime}(u) u_{x}, \quad u_{x t}=-f^{\prime \prime}(u)\left(u_{x}\right)^{2}-f^{\prime}(u) u_{x x}, \\
& u_{t t}=-\left(f^{\prime}(u) u_{t}\right)_{x}=-f^{\prime \prime}(u) u_{x} u_{t}-f^{\prime}(u) u_{x t} .
\end{aligned}
$$

Now the functional $F(u)$ in $(2.10)$ should be understood as $F=\mathcal{F}\left(u, u_{x}, u_{x x}\right)$, though the notation $F(u)$ will still be used for simplicity.

By combining (2.3)-(2.5) and (2.9)-(2.11), we are ready to present the proposed central scheme.

(a.1) Suppose at $t=t^{n}$, the approximations for the first two moments of the solution, denoted as $\left\{\bar{u}_{i}^{n}\right\}$ and $\left\{\bar{v}_{i}^{n}\right\}$, are available on the primal mesh, that is, $\forall i$,

$$
\bar{u}_{i}^{n} \approx \frac{1}{\Delta x} \int_{I_{i}} u\left(x, t^{n}\right) d x, \quad \bar{v}_{i}^{n} \approx \frac{1}{\Delta x} \int_{I_{i}} u\left(x, t^{n}\right) \frac{x-x_{i}}{\Delta x} d x .
$$

Following a staggered central scheme strategy, we compute the first two moments of the solution with respect to the dual mesh at $t=t^{n+1}$, denoted as $\bar{u}_{i+1 / 2}^{n+1}$ and $\bar{v}_{i+1 / 2}^{n+1}$, as follows,

$$
\begin{aligned}
& \bar{u}_{i+1 / 2}^{n+1}=\bar{u}_{i+1 / 2}^{n}-\frac{\Delta t}{\Delta x}\left[F\left(u\left(x_{i+1}, t^{n}\right)\right)-F\left(u\left(x_{i}, t^{n}\right)\right)\right], \\
& \bar{v}_{i+1 / 2}^{n+1}=\bar{v}_{i+1 / 2}^{n}-\frac{\Delta t}{\Delta x}\left[\frac{1}{2}\left[F\left(u\left(x_{i+1}, t^{n}\right)\right)+F\left(u\left(x_{i}, t^{n}\right)\right)\right]-\sum_{l=1}^{4} \omega_{l} F\left(u\left(\mathcal{G}_{l}^{x}, t^{n}\right)\right)\right] .
\end{aligned}
$$

For simplicity of notation, we still use $u$ in all the terms on the right-hand side, though $u$ is no longer the exact solution, and will be reconstructed based on the given data.

(a.2) With $\bar{u}_{i+1 / 2}^{n+1}$ and $\bar{v}_{i+1 / 2}^{n+1}$ being available for any $i$, we further compute the first two moments of the solution with respect to the primal mesh at $t=t^{n+2}$, denoted as $\bar{u}_{i}^{n+2}$ and $\bar{v}_{i}^{n+2}$, as follows,

$$
\begin{aligned}
& \bar{u}_{i}^{n+2}=\bar{u}_{i}^{n+1}-\frac{\Delta t}{\Delta x}\left[F\left(u\left(x_{i+1 / 2}, t^{n+1}\right)\right)-F\left(u\left(x_{i-1 / 2}, t^{n+1}\right)\right)\right], \\
& \bar{v}_{i}^{n+2}=\bar{v}_{i}^{n+1}-\frac{\Delta t}{\Delta x}\left[\frac{1}{2}\left[F\left(u\left(x_{i+1 / 2}, t^{n+1}\right)\right)+F\left(u\left(x_{i-1 / 2}, t^{n+1}\right)\right)\right]-\sum_{l=1}^{4} \omega_{l} F\left(u\left(\hat{\mathcal{G}}_{l}^{x}, t^{n+1}\right)\right)\right] .
\end{aligned}
$$

(a.3) Set $n$ to be $n+2$, and go to (a.1).

Here the Gauss-Lobatto quadrature points associated with $I_{i}$ are $\hat{\mathcal{G}}_{1}^{x}=x_{i-1 / 2}, \hat{\mathcal{G}}_{2}^{x}=x_{i-\frac{\sqrt{5}}{10}}, \hat{\mathcal{G}}_{3}^{x}=x_{i+\frac{\sqrt{5}}{10}}$, and $\hat{\mathcal{G}}_{4}^{x}=x_{i+1 / 2}$. Note that the moments are defined and evolved in a staggered fashion with respect to the discrete time level $n$ on two sets of meshes. The mesh switches back after two time steps.

To finalize the proposed scheme, one needs to reconstruct certain quantities in (a.1) and (a.2). With similarity, we will only focus on (a.1). In this step, to obtain the moments $\bar{u}_{i+1 / 2}^{n+1}$ and $\bar{v}_{i+1 / 2}^{n+1}$ on the dual mesh at the next time $t^{n+1}$ according to (2.13) and (2.14), one will need to reconstruct at the current time $t^{n}, \forall i$, 
(1) the moments $\bar{u}_{i+1 / 2}^{n}, \bar{v}_{i+1 / 2}^{n}$ on the dual mesh;

(2) the point value of $q\left(x_{*}, t^{n}\right)$, where $q=u, u_{x}$, or $u_{x x}$, and $x_{*} \in \mathcal{G}^{x, i}$,

based on the given data $\left\{\bar{u}_{i}^{n}, \bar{v}_{i}^{n}\right\}_{i}$. Recall $F=\mathcal{F}\left(u, u_{x}, u_{x x}\right)$ depends on $u$ and its derivatives $u_{x}, u_{x x}$.

It is also useful to note that

$$
\bar{u}_{i+1 / 2}^{n} \approx \frac{1}{\Delta x} \int_{x_{i}}^{x_{i+1}} u\left(x, t^{n}\right) d x=\frac{1}{\Delta x} \int_{x_{i}}^{x_{i+1 / 2}} u\left(x, t^{n}\right) d x+\frac{1}{\Delta x} \int_{x_{i+1 / 2}}^{x_{i+1}} u\left(x, t^{n}\right) d x .
$$

This implies that to approximate the moment $\bar{u}_{i+1 / 2}^{n}$, one would want to get the half-cell moments: $\frac{1}{\Delta x} \int_{x_{i}}^{x_{i+1 / 2}} u\left(x, t^{n}\right) d x$ and $\frac{1}{\Delta x} \int_{x_{i+1 / 2}}^{x_{i+1}} u\left(x, t^{n}\right) d x$. This is likewise for the moment $\bar{v}_{i+1 / 2}^{n}$,

$$
\bar{v}_{i+1 / 2}^{n} \approx \frac{1}{\Delta x} \int_{x_{i}}^{x_{i+1 / 2}} u\left(x, t^{n}\right) \frac{x-x_{i+1 / 2}}{\Delta x} d x+\frac{1}{\Delta x} \int_{x_{i+1 / 2}}^{x_{i+1}} u\left(x, t^{n}\right) \frac{x-x_{i+1 / 2}}{\Delta x} d x .
$$

To reconstruct the half-cell moments and point values mentioned above, we propose a procedure by adapting the one-dimensional fifth-order accurate Hermite WENO (HWENO) reconstruction of Qiu and Shu [28], where only the function values at the end points of each cell were reconstructed. This reconstruction is not only high-order accurate but also essentially non-oscillatory. Compared with the standard WENO schemes, the stencils in the reconstruction are more compact. In the remaining part of this section, we will describe the reconstruction with great details through steps 1-5, where the reconstruction is based on the two moments $\left\{\bar{u}_{i}^{n}, \bar{v}_{i}^{n}\right\}_{i}$ on the primal mesh at $t=t^{n}$. Since both the data before and after the reconstruction is at the same time level $t^{n}$, the superscript $n$ will be omitted below, together with the dependence on the time $t$ of $u$ and $v$.

Step 1: A HWENO reconstruction of $\frac{1}{\Delta x} \int_{x_{i-1 / 2}}^{x_{i}} u(x) d x$ from the moments $\left\{\bar{u}_{i}, \bar{v}_{i}\right\}_{i}$.

We first introduce three "small" stencils $S_{0}=\left\{I_{i-1}, I_{i}\right\}, S_{1}=\left\{I_{i}, I_{i+1}\right\}, S_{2}=\left\{I_{i-1}, I_{i}, I_{i+1}\right\}$, one "large" stencil $\mathcal{T}=\left\{S_{0}, S_{1}, S_{2}\right\}=S_{2}$, and reconstruct three quadratic Hermite polynomials $p_{0}(x), p_{1}(x), p_{2}(x)$ on $S_{0}, S_{1}, S_{2}$, respectively, and one quartic Hermite polynomial $Q(x)$ on $\mathcal{T}$, satisfying the following conditions,

$$
\begin{gathered}
\frac{1}{\Delta x} \int_{I_{i+j}} p_{0}(x) d x=\bar{u}_{i+j}, \quad j=-1,0, \quad \frac{1}{\Delta x} \int_{I_{i-1}} p_{0}(x) \frac{x-x_{i-1}}{\Delta x} d x=\bar{v}_{i-1}, \\
\frac{1}{\Delta x} \int_{I_{i+j}} p_{1}(x) d x=\bar{u}_{i+j}, \quad j=0,1, \quad \frac{1}{\Delta x} \int_{I_{i+1}} p_{1}(x) \frac{x-x_{i+1}}{\Delta x} d x=\bar{v}_{i+1}, \\
\frac{1}{\Delta x} \int_{I_{i+j}} p_{2}(x) d x=\bar{u}_{i+j}, \quad j=-1,0,1, \\
\frac{1}{\Delta x} \int_{I_{i+j}} Q(x) d x=\bar{u}_{i+j}, \quad j=-1,0,1, \quad \frac{1}{\Delta x} \int_{I_{i+j}} Q(x) \frac{x-x_{i+j}}{\Delta x} d x=\bar{v}_{i+j}, \quad j=-1,1 .
\end{gathered}
$$

To reconstruct $\frac{1}{\Delta x} \int_{x_{i-1 / 2}}^{x_{i}} u(x) d x$, we further compute the half-cell zeroth-order moments, which are also averages, of these polynomials over $\left[x_{i-1 / 2}, x_{i}\right]$, and the results can be given explicitly in terms of the moments $\left\{\bar{u}_{i}, \bar{v}_{i}\right\}_{i}$,

$$
\begin{aligned}
& \frac{1}{\Delta x} \int_{x_{i-1 / 2}}^{x_{i}} p_{0}(x) d x=\frac{1}{4} \bar{u}_{i-1}+\frac{1}{4} \bar{u}_{i}+\frac{3}{2} \bar{v}_{i-1}, \\
& \frac{1}{\Delta x} \int_{x_{i-1 / 2}}^{x_{i}} p_{1}(x) d x=\frac{3}{4} \bar{u}_{i}-\frac{1}{4} \bar{u}_{i+1}+\frac{3}{2} \bar{v}_{i+1}, \\
& \frac{1}{\Delta x} \int_{x_{i-1 / 2}}^{x_{i}} p_{2}(x) d x=\frac{1}{16} \bar{u}_{i-1}+\frac{1}{2} \bar{u}_{i}-\frac{1}{16} \bar{u}_{i+1},
\end{aligned}
$$




$$
\frac{1}{\Delta x} \int_{x_{i-1 / 2}}^{x_{i}} Q(x) d x=\frac{121}{1216} \bar{u}_{i-1}+\frac{1}{2} \bar{u}_{i}-\frac{121}{1216} \bar{u}_{i+1}+\frac{135}{304} \bar{v}_{i-1}+\frac{135}{304} \bar{v}_{i+1} .
$$

Now one can find the linear weights, denoted as $\gamma_{0}, \gamma_{1}$ and $\gamma_{2}$, such that

$$
\frac{1}{\Delta x} \int_{x_{i-1 / 2}}^{x_{i}} Q(x) d x=\frac{1}{\Delta x} \sum_{j=0}^{2} \gamma_{j} \int_{x_{i-1 / 2}}^{x_{i}} p_{j}(x) d x
$$

holds when $\left\{\bar{u}_{i}, \bar{v}_{i}\right\}_{i}$ take arbitrary values over the stencil $\mathcal{T}$. This requirement leads to

$$
\gamma_{0}=\frac{45}{152}, \quad \gamma_{1}=\frac{45}{152}, \quad \gamma_{2}=\frac{31}{76} .
$$

With the linear weights, we combine the relatively lower order approximations, which are third-order accurate here, into a higher order approximation that is fifth-order accurate in our case.

An important ingredient of WENO-type methods for solving hyperbolic conservation laws with strong shocks or other discontinuities in the solutions is nonlinear weights, which are applied to control spurious oscillations. To obtain the nonlinear weights, we compute a smoothness indicator $\beta_{j}$ for each stencil $S_{j}$ $(j=0,1,2)$, which measures how smooth the function $p_{j}(x)$ is in the target cell $I_{i}$. The smaller the smoothness indicator $\beta_{j}$ is, the smoother the function $p_{j}(x)$ is in the cell $I_{i}$. Following [15], the smoothness indicator $\beta_{j}$ is defined as below,

$$
\beta_{j}=\sum_{l=1}^{2} \int_{I_{i}} \Delta x^{2 l-1}\left(\frac{\partial^{l}}{\partial x^{l}} p_{j}(x)\right)^{2} d x,
$$

and it can be further given explicitly in terms of the moments of the solution for the convenience of the actual implementation,

$$
\left\{\begin{array}{l}
\beta_{0}=4\left(\bar{u}_{i-1}-\bar{u}_{i}+6 \bar{v}_{i-1}\right)^{2}+\frac{13}{3}\left(\bar{u}_{i-1}-\bar{u}_{i}+12 \bar{v}_{i-1}\right)^{2}, \\
\beta_{1}=4\left(\bar{u}_{i}-\bar{u}_{i+1}+6 \bar{v}_{i+1}\right)^{2}+\frac{13}{3}\left(\bar{u}_{i}-\bar{u}_{i+1}+12 \bar{v}_{i+1}\right)^{2}, \\
\beta_{2}=\frac{1}{4}\left(\bar{u}_{i-1}-\bar{u}_{i+1}\right)^{2}+\frac{13}{12}\left(\bar{u}_{i-1}-2 \bar{u}_{i}+\bar{u}_{i+1}\right)^{2} .
\end{array}\right.
$$

With the smoothness indicators $\left\{\beta_{j}\right\}_{j}$ in (2.19) and the linear weights $\left\{\gamma_{j}\right\}_{j}$ in (2.17), we can now compute the nonlinear weights $\omega_{j}, j=0,1,2$,

$$
\omega_{j}=\frac{\bar{\omega}_{j}}{\sum_{k=0}^{2} \bar{\omega}_{k}}, \quad \text { where } \bar{\omega}_{k}=\frac{\gamma_{k}}{\left(\varepsilon+\beta_{k}\right)^{2}}, \quad k=0,1,2 .
$$

Here $\varepsilon>0$ is a small constant to avoid the denominator to be zero. We use $\varepsilon=10^{-6}$ in all numerical examples in this paper.

Finally, a fifth-order HWENO approximation for $\frac{1}{\Delta x} \int_{x_{i-1 / 2}}^{x_{i}} u(x) d x$ is given as

$$
\frac{1}{\Delta x} \int_{x_{i-1 / 2}}^{x_{i}} u(x) d x \approx \frac{1}{\Delta x} \sum_{j=0}^{2} \omega_{j} \int_{x_{i-1 / 2}}^{x_{i}} p_{j}(x) d x,
$$

where $\frac{1}{\Delta x} \int_{x_{i-1 / 2}}^{x_{i}} p_{j}(x) d x, j=0,1,2$ are given in (2.16).

In the target cell $I_{i}$, the right half-cell average of $u$ over $\left[x_{i}, x_{i+1 / 2}\right]$ can be approximated based on the local conservation of $u$,

$$
\frac{1}{\Delta x} \int_{x_{i}}^{x_{i+1 / 2}} u(x) d x \approx \bar{u}_{i}-\frac{1}{\Delta x} \int_{x_{i-1 / 2}}^{x_{i}} u(x) d x .
$$


Step 2. A HWENO reconstruction of $\frac{1}{\Delta x} \int_{x_{i-1 / 2}}^{x_{i}} u(x) \frac{x-x_{i-1 / 2}}{\Delta x} d x$ from the moments $\left\{\bar{u}_{i}, \bar{v}_{i}\right\}_{i}$.

In this step, the same stencils $S_{0}, S_{1}, S_{2}, \mathcal{T}$ as in step 1 are used. We reconstruct three cubic Hermite polynomials $p_{0}(x), p_{1}(x), p_{2}(x)$ on $S_{0}, S_{1}, S_{2}$, respectively, and one quintic Hermite polynomial $Q(x)$ on $\mathcal{T}$, satisfying

$$
\begin{aligned}
& \frac{1}{\Delta x} \int_{I_{i+j}} p_{0}(x) d x=\bar{u}_{i+j}, \quad \frac{1}{\Delta x} \int_{I_{i+j}} p_{0}(x) \frac{x-x_{i+j}}{\Delta x} d x=\bar{v}_{i+j}, \quad j=-1,0, \\
& \frac{1}{\Delta x} \int_{I_{i+j}} p_{1}(x) d x=\bar{u}_{i+j}, \quad \frac{1}{\Delta x} \int_{I_{i+j}} p_{1}(x) \frac{x-x_{i+j}}{\Delta x} d x=\bar{v}_{i+j}, \quad j=0,1, \\
& \frac{1}{\Delta x} \int_{I_{i+j}} p_{2}(x) d x=\bar{u}_{i+j}, \quad j=-1,0,1, \quad \frac{1}{\Delta x} \int_{I_{i}} p_{2}(x) \frac{x-x_{i}}{\Delta x} d x=\bar{v}_{i}, \\
& \frac{1}{\Delta x} \int_{I_{i+j}} Q(x) d x=\bar{u}_{i+j}, \quad \frac{1}{\Delta x} \int_{I_{i+j}} Q(x) \frac{x-x_{i+j}}{\Delta x} d x=\bar{v}_{i+j}, \quad j=-1,0,1 .
\end{aligned}
$$

To reconstruct $\frac{1}{\Delta x} \int_{x_{i-1 / 2}}^{x_{i}} u(x) \frac{x-x_{i-1 / 2}}{\Delta x} d x$, we further compute the half-cell first-order moments of these polynomials over $\left[x_{i-1 / 2}, x_{i}\right]$, and the results can be given explicitly in terms of $\left\{\bar{u}_{i}, \bar{v}_{i}\right\}_{i}$,

$$
\begin{gathered}
\frac{1}{\Delta x} \int_{x_{i-1 / 2}}^{x_{i}} p_{0}(x) \frac{x-x_{i-1 / 2}}{\Delta x} d x=-\frac{1}{64} \bar{u}_{i-1}+\frac{9}{64} \bar{u}_{i}-\frac{1}{16} \bar{v}_{i-1}-\frac{3}{8} \bar{v}_{i} \\
\frac{1}{\Delta x} \int_{x_{i-1 / 2}}^{x_{i}} p_{1}(x) \frac{x-x_{i-1 / 2}}{\Delta x} d x=\frac{19}{128} \bar{u}_{i}-\frac{3}{128} \bar{u}_{i+1}-\frac{5}{64} \bar{v}_{i}+\frac{7}{64} \bar{v}_{i+1} \\
\frac{1}{\Delta x} \int_{x_{i-1 / 2}}^{x_{i}} p_{2}(x) \frac{x-x_{i-1 / 2}}{\Delta x} d x=-\frac{7}{2112} \bar{u}_{i-1}+\frac{25}{192} \bar{u}_{i}-\frac{1}{528} \bar{u}_{i+1}-\frac{47}{176} \bar{v}_{i} \\
\frac{1}{\Delta x} \int_{x_{i-1 / 2}}^{x_{i}} Q(x) \frac{x-x_{i-1 / 2}}{\Delta x} d x=-\frac{5}{512} \bar{u}_{i-1}+\frac{71}{512} \bar{u}_{i}-\frac{1}{256} \bar{u}_{i+1}-\frac{9}{256} \bar{v}_{i-1}-\frac{77}{256} \bar{v}_{i}+\frac{1}{64} \bar{v}_{i+1} .
\end{gathered}
$$

Just as in step 1 , we next want to obtain the nonlinear weights $\omega_{j}, j=0,1,2$, with which, a HWENO reconstruction can be given for $\frac{1}{\Delta x} \int_{x_{i-1 / 2}}^{x_{i}} u(x) \frac{x-x_{i-1 / 2}}{\Delta x} d x$. To achieve this, we first find the linear weights, denoted as $\gamma_{0}^{\prime}, \gamma_{1}^{\prime}$ and $\gamma_{2}^{\prime}$, such that

$$
\frac{1}{\Delta x} \int_{x_{i-1 / 2}}^{x_{i}} Q(x) \frac{x-x_{i-1 / 2}}{\Delta x} d x=\frac{1}{\Delta x} \sum_{j=0}^{2} \gamma_{j}^{\prime} \int_{x_{i-1 / 2}}^{x_{i}} p_{j}(x) \frac{x-x_{i-1 / 2}}{\Delta x} d x
$$

holds when $\left\{\bar{u}_{i}, \bar{v}_{i}\right\}_{i}$ take arbitrary values over the stencil $\mathcal{T}$, and this leads to

$$
\gamma_{0}^{\prime}=\frac{9}{16}, \quad \gamma_{1}^{\prime}=\frac{1}{7}, \quad \gamma_{2}^{\prime}=\frac{33}{112}
$$

We then compute the smoothness indicators $\beta_{j}, j=0,1,2$ as below

$$
\beta_{j}=\sum_{l=2}^{3} \int_{I_{i}} \Delta x^{2 l-1}\left(\frac{\partial^{l}}{\partial x^{l}} p_{j}(x)\right)^{2} d x
$$

Since we are reconstructing the first-order moment rather than the solution itself, the summation starts from the second derivative to the third derivative of the cubic polynomial $p_{j}(x)$. For the convenience of the actual implementation, the smoothness indicators $\left\{\beta_{j}\right\}_{j=0}^{2}$ can be further given in quadratic forms of the moments 
of the solution,

$$
\left\{\begin{array}{l}
\beta_{0}=\frac{1}{4}\left(15 \bar{u}_{i-1}-15 \bar{u}_{i}+66 \bar{v}_{i-1}+114 \bar{v}_{i}\right)^{2}+\frac{975}{4}\left(\bar{u}_{i-1}-\bar{u}_{i}+6 \bar{v}_{i-1}+6 \bar{v}_{i}\right)^{2} \\
\beta_{1}=\frac{1}{4}\left(15 \bar{u}_{i}-15 \bar{u}_{i+1}+114 \bar{v}_{i}+66 \bar{v}_{i+1}\right)^{2}+\frac{975}{4}\left(\bar{u}_{i}-\bar{u}_{i+1}+6 \bar{v}_{i}+6 \bar{v}_{i+1}\right)^{2} \\
\beta_{2}=\left(\bar{u}_{i-1}-2 \bar{u}_{i}+\bar{u}_{i+1}\right)^{2}+\frac{975}{121}\left(\bar{u}_{i-1}-\bar{u}_{i+1}+24 \bar{v}_{i}\right)^{2} .
\end{array}\right.
$$

With the smoothness indicators $\left\{\beta_{j}\right\}_{j}$ in $(2.21)$ and the linear weights $\left\{\gamma_{j}^{\prime}\right\}_{j}$ in $(2.20)$, the nonlinear weights $\omega_{j}, j=0,1,2$, can be computed,

$$
\omega_{j}=\frac{\bar{\omega}_{j}}{\sum_{k=0}^{2} \bar{\omega}_{k}}, \quad \text { where } \bar{\omega}_{k}=\frac{\gamma_{k}^{\prime}}{\left(\varepsilon+\beta_{k}\right)^{2}}, \quad k=0,1,2
$$

Finally, the HWENO approximation for $\frac{1}{\Delta x} \int_{x_{i-1 / 2}}^{x_{i}} u(x) \frac{x-x_{i-1 / 2}}{\Delta x} d x$ is given as

$$
\frac{1}{\Delta x} \int_{x_{i-1 / 2}}^{x_{i}} u(x) \frac{x-x_{i-1 / 2}}{\Delta x} d x \approx \frac{1}{\Delta x} \sum_{j=0}^{2} \omega_{j} \int_{x_{i-1 / 2}}^{x_{i}} p_{j}(x) \frac{x-x_{i-1 / 2}}{\Delta x} d x .
$$

In the target cell $I_{i}$, the right half first-order moment $\frac{1}{\Delta x} \int_{x_{i}}^{x_{i+1 / 2}} u(x) \frac{x-x_{i+1 / 2}}{\Delta x} d x$ can be reconstructed similarly as the above procedure.

Step 3. A HWENO reconstruction of $u\left(x_{i}\right)$ from the moments $\left\{\bar{u}_{i}, \bar{v}_{i}\right\}_{i}$.

In this step, the same stencils $S_{0}, S_{1}, S_{2}, \mathcal{T}$ as in step 1 are used, together with the same constructed polynomials $p_{0}(x), p_{1}(x), p_{2}(x)$ and $Q(x)$ in $(2.15)$. The point value of these polynomials at $x_{i}$ can be expressed in terms of the moments,

$$
\begin{gathered}
p_{0}\left(x_{i}\right)=\frac{1}{12} \bar{u}_{i-1}+\frac{11}{12} \bar{u}_{i}+\bar{v}_{i-1}, \\
p_{1}\left(x_{i}\right)=\frac{11}{12} \bar{u}_{i}+\frac{1}{12} \bar{u}_{i+1}-\bar{v}_{i+1}, \\
p_{2}\left(x_{i}\right)=-\frac{1}{24} \bar{u}_{i-1}+\frac{13}{12} \bar{u}_{i}-\frac{1}{24} \bar{u}_{i+1}, \\
Q\left(x_{i}\right)=-\frac{43}{384} \bar{u}_{i-1}+\frac{235}{192} \bar{u}_{i}-\frac{43}{384} \bar{u}_{i+1}-\frac{27}{64} \bar{v}_{i-1}+\frac{27}{64} \bar{v}_{i+1} .
\end{gathered}
$$

The linear weights $\gamma_{0}, \gamma_{1}, \gamma_{2}$ are chosen such that

$$
Q\left(x_{i}\right)=\sum_{j=0}^{2} \gamma_{j} p_{j}\left(x_{i}\right)
$$

holds when $\left\{\bar{u}_{i}, \bar{v}_{i}\right\}_{i}$ take arbitrary values over the stencil $\mathcal{T}$, and this requirement leads to

$$
\gamma_{0}=-\frac{27}{64}, \quad \gamma_{1}=-\frac{27}{64}, \quad \gamma_{2}=\frac{59}{32}
$$

Note that two linear weights are negative in (2.22), and the corresponding WENO-type approximation with such weights can be unstable. To avoid this, we follow the splitting technique developed in [30] to handle negative weights in WENO schemes. More specifically, we split the linear weights into two groups

$$
\tilde{\gamma}_{j}^{+}=\frac{1}{2}\left(\gamma_{j}+3\left|\gamma_{j}\right|\right), \quad \tilde{\gamma}_{j}^{-}=\tilde{\gamma}_{j}^{+}-\gamma_{j}, \quad j=0,1,2
$$


which can be shown to satisfy

$$
\tilde{\gamma}_{j}^{+}=2 \gamma_{j}, \tilde{\gamma}_{j}^{-}=\gamma_{j}, \quad \text { for } \gamma_{j}>0 ; \quad \tilde{\gamma}_{j}^{+}=-\gamma_{j}, \tilde{\gamma}_{j}^{-}=-2 \gamma_{j}, \quad \text { for } \gamma_{j}<0 .
$$

We further scale the terms by introducing

$$
\sigma^{ \pm}=\sum_{j=0}^{2} \tilde{\gamma}_{j}^{ \pm}, \quad \gamma_{j}^{ \pm}=\tilde{\gamma}_{j}^{ \pm} / \sigma^{ \pm}, \quad j=0,1,2 .
$$

For the linear weights in $(2.22)$, this gives

$$
\begin{gathered}
\sigma^{+}=\frac{145}{32}, \quad \sigma^{-}=\frac{113}{32}, \\
\gamma_{0}^{+}=\frac{27}{290}, \gamma_{1}^{+}=\frac{27}{290}, \gamma_{2}^{+}=\frac{118}{145}, \quad \gamma_{0}^{-}=\frac{27}{113}, \gamma_{1}^{-}=\frac{27}{113}, \gamma_{2}^{-}=\frac{59}{113} .
\end{gathered}
$$

The HWENO reconstruction will be performed for each group by computing the nonlinear weights $\omega_{j}^{+}$ and $\omega_{j}^{-}, j=0,1,2$ separately, with the same smoothness indicators $\left\{\beta_{j}\right\}_{j}$ as in (2.19),

$$
\omega_{j}^{ \pm}=\frac{\bar{\omega}_{j}^{ \pm}}{\sum_{k=0}^{2} \bar{\omega}_{k}^{ \pm}}, \quad \text { where } \bar{\omega}_{k}^{ \pm}=\frac{\gamma_{k}^{ \pm}}{\left(\varepsilon+\beta_{k}\right)^{2}}, \quad k=0,1,2 .
$$

The final HWENO reconstruction of $u\left(x_{i}\right)$ is now taken as a combination of the reconstructions using the two groups of weights

$$
u\left(x_{i}\right) \approx \sigma^{+} \sum_{j=0}^{2} \omega_{j}^{+} p_{j}\left(x_{i}\right)-\sigma^{-} \sum_{j=0}^{2} \omega_{j}^{-} p_{j}\left(x_{i}\right) .
$$

In the adopted technique to treat negative linear weights, the key is to ensure that every stencil has a significant representation in both positive and negative weight groups. Within each group, one still follows the standard HWENO idea of redistributing the weights subject to a fixed sum according to the smoothness of the approximations.

The reconstructions of the other two point values $u\left(x_{*}\right)$ with $x_{*} \in \mathcal{G}^{x, i}$ are similar to the above procedure. Fortunately, the linear weights in the reconstruction of $u$ at the other two points are all positive, based on which the nonlinear weights can be constructed directly without the splitting technique.

Step 4. A HWENO reconstruction of $u_{x}\left(x_{i}\right)$ from the moments $\left\{\bar{u}_{i}, \bar{v}_{i}\right\}_{i}$.

In this step, we use the same stencils $S_{0}, S_{1}, S_{2}, \mathcal{T}$ and the same constructed polynomials $p_{0}(x), p_{1}(x)$, $p_{2}(x), Q(x)$ as in step 2. The point values of the first derivative of these polynomials at $x_{i}$ can be expressed in terms of the moments,

$$
\begin{gathered}
p_{0}^{\prime}\left(x_{i}\right)=\frac{1}{\Delta x}\left(-\frac{3}{8} \bar{u}_{i-1}+\frac{3}{8} \bar{u}_{i}-\frac{9}{4} \bar{v}_{i-1}+\frac{39}{4} \bar{v}_{i}\right) \\
p_{1}^{\prime}\left(x_{i}\right)=\frac{1}{\Delta x}\left(-\frac{3}{8} \bar{u}_{i}+\frac{3}{8} \bar{u}_{i+1}+\frac{39}{4} \bar{v}_{i}-\frac{9}{4} \bar{v}_{i+1}\right) \\
p_{2}^{\prime}\left(x_{i}\right)=\frac{1}{\Delta x}\left(\frac{3}{44} \bar{u}_{i-1}-\frac{3}{44} \bar{u}_{i+1}+\frac{150}{11} \bar{v}_{i}\right) \\
Q^{\prime}\left(x_{i}\right)=\frac{1}{\Delta x}\left(\frac{167}{576} \bar{u}_{i-1}-\frac{167}{576} \bar{u}_{i+1}+\frac{281}{288} \bar{v}_{i-1}+\frac{2449}{144} \bar{v}_{i}+\frac{281}{288} \bar{v}_{i+1}\right) .
\end{gathered}
$$


The linear weights $\gamma_{0}^{\prime}, \gamma_{1}^{\prime}, \gamma_{2}^{\prime}$ can be determined by requiring

$$
Q^{\prime}\left(x_{i}\right)=\sum_{j=0}^{2} \gamma_{j}^{\prime} p_{j}^{\prime}\left(x_{i}\right)
$$

hold when $\left\{\bar{u}_{i}, \bar{v}_{i}\right\}_{i}$ take arbitrary values over the stencil $\mathcal{T}$, and this leads to

$$
\gamma_{0}^{\prime}=-\frac{281}{648}, \quad \gamma_{1}^{\prime}=-\frac{281}{648}, \quad \gamma_{2}^{\prime}=\frac{605}{324} .
$$

There are two negative linear weights in (2.23). Following the procedure provided in step 3 , we get

$$
\begin{gathered}
\sigma^{\prime+}=\frac{1491}{324}, \quad \sigma^{\prime-}=\frac{1167}{324}, \\
\gamma_{0}^{\prime+}=\frac{281}{2982}, \gamma_{1}^{\prime+}=\frac{281}{2982}, \gamma_{2}^{\prime+}=\frac{1210}{1491}, \quad \gamma_{0}^{\prime-}=\frac{281}{1167}, \gamma_{1}^{\prime-}=\frac{281}{1167}, \gamma_{2}^{\prime-}=\frac{605}{1167} .
\end{gathered}
$$

The HWENO reconstruction will be performed for each group by computing the nonlinear weights $\omega_{j}^{+}$ and $\omega_{j}^{-}, j=0,1,2$ separately, with the same smoothness indicators $\left\{\beta_{j}\right\}_{j}$ as in $(2.21)$,

$$
\omega_{j}^{ \pm}=\frac{\bar{\omega}_{j}^{ \pm}}{\sum_{k=0}^{2} \bar{\omega}_{k}^{ \pm}}, \quad \text { where } \bar{\omega}_{k}^{ \pm}=\frac{\gamma_{k}^{\prime \pm}}{\left(\varepsilon+\beta_{k}\right)^{2}}, \quad k=0,1,2 .
$$

The final HWENO reconstruction of $u_{x}\left(x_{i}\right)$ is now obtained as

$$
u_{x}\left(x_{i}\right) \approx \sigma^{\prime+} \sum_{j=0}^{2} \omega_{j}^{+} p_{j}^{\prime}\left(x_{i}\right)-\sigma^{\prime-} \sum_{j=0}^{2} \omega_{j}^{-} p_{j}^{\prime}\left(x_{i}\right) .
$$

The reconstructions of the other two point values $u_{x}\left(x_{*}\right)$ with $x_{*} \in \mathcal{G}^{x, i}$ are similar to the above procedure, with the related linear weights being all positive.

Step 5. A HWENO reconstruction of $u_{x x}\left(x_{i}\right)$ from the moments $\left\{\bar{u}_{i}, \bar{v}_{i}\right\}_{i}$.

In this step, we use the same stencils $S_{0}, S_{1}, S_{2}, \mathcal{T}$ and the same constructed polynomials $p_{0}(x), p_{1}(x)$, $p_{2}(x), Q(x)$ as in step 2 . The point values of the second derivative of these polynomials at $x_{i}$ can be expressed in terms of the moments,

$$
\begin{gathered}
p_{0}^{\prime \prime}\left(x_{i}\right)=\frac{1}{\Delta x^{2}}\left(\frac{15}{2} \bar{u}_{i-1}-\frac{15}{2} \bar{u}_{i}+33 \bar{v}_{i-1}+57 \bar{v}_{i}\right), \\
p_{1}^{\prime \prime}\left(x_{i}\right)=\frac{1}{\Delta x^{2}}\left(-\frac{15}{2} \bar{u}_{i}+\frac{15}{2} \bar{u}_{i+1}-57 \bar{v}_{i}-33 \bar{v}_{i+1}\right), \\
p_{2}^{\prime \prime}\left(x_{i}\right)=\frac{1}{\Delta x^{2}}\left(\bar{u}_{i-1}-2 \bar{u}_{i}+\bar{u}_{i+1}\right), \\
Q^{\prime \prime}\left(x_{i}\right)=\frac{1}{\Delta x^{2}}\left(\frac{23}{8} \bar{u}_{i-1}-\frac{23}{4} \bar{u}_{i}+\frac{23}{8} \bar{u}_{i+1}+\frac{45}{4} \bar{v}_{i-1}-\frac{45}{4} \bar{v}_{i+1}\right) .
\end{gathered}
$$

The linear weights $\gamma_{0}^{\prime \prime}, \gamma_{1}^{\prime \prime}, \gamma_{2}^{\prime \prime}$ are determined by requiring

$$
Q^{\prime \prime}\left(x_{i}\right)=\sum_{j=0}^{2} \gamma_{j}^{\prime \prime} p_{j}^{\prime \prime}\left(x_{i}\right)
$$

hold when $\left\{\bar{u}_{i}, \bar{v}_{i}\right\}_{i}$ take arbitrary values over the stencil $\mathcal{T}$, and this leads to

$$
\gamma_{0}^{\prime \prime}=\frac{15}{44}, \quad \gamma_{1}^{\prime \prime}=\frac{15}{44}, \quad \gamma_{2}^{\prime \prime}=\frac{7}{22} .
$$


We then compute the smoothness indicators $\beta_{j}, j=0,1,2$ as below

$$
\beta_{j}=\Delta x^{5} \int_{I_{i}}\left(\frac{\partial^{3}}{\partial x^{3}} p_{j}(x)\right)^{2} d x .
$$

Since we are reconstructing the second derivative rather than the solution itself, the smoothness indicator $\beta_{j}$ only depends on the third derivative of the cubic polynomial $p_{j}(x)$. For the convenience of the actual implementation, the smoothness indicators $\left\{\beta_{j}\right\}_{j=0}^{2}$ can be further given in terms of the moments,

$$
\left\{\begin{array}{l}
\beta_{0}=225\left(\bar{u}_{i-1}-\bar{u}_{i}+6\left(\bar{v}_{i-1}+\bar{v}_{i}\right)\right)^{2}, \\
\beta_{1}=225\left(\bar{u}_{i}-\bar{u}_{i+1}+6\left(\bar{v}_{i}+\bar{v}_{i+1}\right)\right)^{2} \\
\beta_{2}=\frac{900}{121}\left(\bar{u}_{i-1}-\bar{u}_{i+1}+24 \bar{v}_{i}\right)^{2} .
\end{array}\right.
$$

With the smoothness indicators $\left\{\beta_{j}\right\}_{j}$ in (2.25) and the linear weights in (2.24), we can compute the nonlinear weights $\omega_{j}, j=0,1,2$,

$$
\omega_{j}=\frac{\bar{\omega}_{j}}{\sum_{k=0}^{2} \bar{\omega}_{k}}, \quad \text { where } \bar{\omega}_{k}=\frac{\gamma_{k}^{\prime \prime}}{\left(\varepsilon+\beta_{k}\right)^{2}}, \quad k=0,1,2
$$

The final HWENO reconstruction for $u_{x x}\left(x_{i}\right)$ is now obtained as

$$
u_{x x}\left(x_{i}\right) \approx \sum_{j=0}^{2} \omega_{j} p_{j}^{\prime \prime}\left(x_{i}\right) .
$$

The reconstructions of the other two point values $u_{x x}\left(x_{*}\right)$ with $x_{*} \in \mathcal{G}^{x, i}$ are similar to the above procedure. Some negative linear weights will appear in these reconstructions, and the technique described in step 3 is adopted to treat the negative weights.

Remark 1. Following the current central scheme framework, in the equations (2.13) and (2.14) to update $\bar{u}_{i+1 / 2}^{n+1}$ and $\bar{v}_{i+1 / 2}^{n+1}$, the flux functions $F$ are evaluated at $x_{*}$ with $x_{*} \in \mathcal{G}^{x, i}$ and the time $t^{n}$. This requires the reconstructions of the point values of $u, u_{x}, u_{x x}$ at $x_{*}$ based on moments $\left\{\bar{u}_{i}^{n}, \bar{v}_{i}^{n}\right\}_{i}$ at the same time level. Since all the related reconstructions (see steps 3-5) involve stencils including the cell $I_{i}$, and $x_{*}$ are interior points, these reconstructed point values are naturally single-valued. Therefore unlike in upwind type methods, there is no need to use numerical fluxes for $F$.

Remark 2. The scheme also works if a linear reconstruction is used in step 5, and this can reduce the computational cost. However, the reconstructions proposed in this paper lead to better numerical results for complex examples such as the forward step problem. One can refer to [35] for more details.

Remark 3. In steps 2, 4, 5, if we instead use the same constructed quadratic Hermite polynomials $p_{0}(x)$, $p_{1}(x), p_{2}(x)$ and a quartic Hermite polynomial $Q(x)$ as in step 1, the resulting scheme works well for most examples without the degeneracy of the accuracy. Our numerical experiments, however, show that with the current reconstruction strategy which involves cubic Hermite polynomials $p_{0}(x), p_{1}(x), p_{2}(x)$ and a quintic Hermite polynomial $Q(x)$ in steps 2, 4, 5, the scheme overall performs better. One can refer to [35] for more details.

Finally in this section, we will briefly discuss the system case. For the systems of conservation laws, such as the Euler equations of gas dynamics, each of the HWENO reconstructions in steps 1-5 can be performed 
for the unknown solution component by component. Alternatively this can be performed based on the local characteristic decomposition, which often provides better performance of the schemes yet computationally is more costly. In order to effectively control the spurious oscillations therefore to enhance the numerical stability while maintaining good computational efficiency, we follow the idea in $[26,34]$ : we apply the local characteristic decomposition during the reconstructions of half-cell moments in steps 1-2 based on $\left\{\bar{u}_{i}, \bar{v}_{i}\right\}_{i}$. All the remaining reconstructions, which are for point values, are still implemented through a componentwise procedure.

From the method formulation point of view, when one converts the second and higher order time derivatives to spatial derivatives in the system case, one needs to work with terms such as $f^{\prime}(u)$ (a Jacobian matrix) and $f^{\prime \prime}(u)$ (a 3D "matrix", a tensor). This can become very involved. We have used MATLAB Symbolic Math Toolbox to assist with the derivation of the proposed methods.

\subsection{Two-dimensional case}

Consider the two-dimensional scalar conservation law

$$
\left\{\begin{array}{l}
u_{t}+f(u)_{x}+g(u)_{y}=0, \\
u(x, y, 0)=u_{0}(x, y) .
\end{array}\right.
$$

The proposed numerical method will be defined on staggered meshes. For simplicity of presentation, uniform meshes are used with the meshsizes $\Delta x$ in the $x$ direction, and $\Delta y$ in the $y$ direction. Each cell of the primal mesh is denoted as $I_{i j}=\left[x_{i-1 / 2}, x_{i+1 / 2}\right] \times\left[y_{j-1 / 2}, y_{j+1 / 2}\right]$ with its cell center $\left(x_{i}, y_{j}\right)$; and each cell of the dual mesh is denoted as $I_{i+1 / 2, j+1 / 2}=\left[x_{i}, x_{i+1}\right] \times\left[y_{j}, y_{j+1}\right]$ with its cell center $\left(x_{i+1 / 2}, y_{j+1 / 2}\right)$.

We multiply $\frac{x-x_{c}}{\Delta x}, \frac{y-y_{c}}{\Delta y}$, and $\frac{x-x_{c}}{\Delta x} \frac{y-y_{c}}{\Delta y}$, which are locally scaled linearly independent polynomials of total degree one, to (2.26), and obtain the equations for the first-order moments of the solution

$$
\left\{\begin{array}{l}
u_{t} \frac{x-x_{c}}{\Delta x}+\left(f(u)_{x}+g(u)_{y}\right) \frac{x-x_{c}}{\Delta x}=0 \\
u_{t} \frac{y-y_{c}}{\Delta y}+\left(f(u)_{x}+g(u)_{y}\right) \frac{y-y_{c}}{\Delta y}=0 \\
u_{t} \frac{x-x_{c}}{\Delta x} \frac{y-y_{c}}{\Delta y}+\left(f(u)_{x}+g(u)_{y}\right) \frac{x-x_{c}}{\Delta x} \frac{y-y_{c}}{\Delta y}=0
\end{array}\right.
$$

where $\left(x_{c}, y_{c}\right)$ is the center of the relevant mesh element. In particular, for a cell $I_{i j}$ from the primal mesh, we will take $\left(x_{c}, y_{c}\right)=\left(x_{i}, y_{j}\right)$; and for a cell $I_{i+1 / 2, j+1 / 2}$ from the dual mesh, we will take $\left(x_{c}, y_{c}\right)=$ $\left(x_{i+1 / 2}, y_{j+1 / 2}\right)$.

Next we proceed to derive our proposed method by utilizing the governing equation (2.26) as well as the new equations in (2.27). We integrate these equations over $I_{i+1 / 2, j+1 / 2} \times\left[t^{n}, t^{n+1}\right]$, apply integration by parts, and get

$$
\begin{aligned}
\frac{1}{\Delta x \Delta y} \int_{I_{i+1 / 2, j+1 / 2}} u\left(x, y, t^{n+1}\right) d x d y=\frac{1}{\Delta x \Delta y} \int_{I_{i+1 / 2, j+1 / 2}} u\left(x, y, t^{n}\right) d x d y \\
-\frac{1}{\Delta x \Delta y} \int_{t^{n}}^{t^{n+1}} \int_{y_{j}}^{y_{j+1}}\left[f\left(u\left(x_{i+1}, y, t\right)\right)-f\left(u\left(x_{i}, y, t\right)\right)\right] d y d t \\
-\frac{1}{\Delta x \Delta y} \int_{t^{n}}^{t^{n+1}} \int_{x_{i}}^{x_{i+1}}\left[g\left(u\left(x, y_{j+1}, t\right)\right)-g\left(u\left(x, y_{j}, t\right)\right)\right] d x d t
\end{aligned}
$$




$$
\begin{aligned}
& \frac{1}{\Delta x \Delta y} \int_{I_{i+1 / 2, j+1 / 2}} u\left(x, y, t^{n+1}\right) \frac{x-x_{i+1 / 2}}{\Delta x} d x d y=\frac{1}{\Delta x \Delta y} \int_{I_{i+1 / 2, j+1 / 2}} u\left(x, y, t^{n}\right) \frac{x-x_{i+1 / 2}}{\Delta x} d x d y \\
& -\frac{1}{2 \Delta x \Delta y} \int_{t^{n}}^{t^{n+1}} \int_{y_{j}}^{y_{j+1}}\left[f\left(u\left(x_{i+1}, y, t\right)\right)+f\left(u\left(x_{i}, y, t\right)\right)\right] d y d t \\
& +\frac{1}{\Delta x^{2} \Delta y} \int_{t^{n}}^{t^{n+1}} \int_{x_{i}}^{x_{i+1}} \int_{y_{j}}^{y_{j+1}} f(u(x, y, t)) d x d y d t \\
& -\frac{1}{\Delta x \Delta y} \int_{t^{n}}^{t^{n+1}} \int_{x_{i}}^{x_{i+1}}\left[g\left(u\left(x, y_{j+1}, t\right)\right)-g\left(u\left(x, y_{j}, t\right)\right)\right] \frac{x-x_{i+1 / 2}}{\Delta x} d x d t, \\
& \frac{1}{\Delta x \Delta y} \int_{I_{i+1 / 2, j+1 / 2}} u\left(x, y, t^{n+1}\right) \frac{y-y_{j+1 / 2}}{\Delta y} d x d y=\frac{1}{\Delta x \Delta y} \int_{I_{i+1 / 2, j+1 / 2}} u\left(x, y, t^{n}\right) \frac{y-y_{j+1 / 2}}{\Delta y} d x d y \\
& -\frac{1}{\Delta x \Delta y} \int_{t^{n}}^{t^{n+1}} \int_{y_{j}}^{y_{j+1}}\left[f\left(u\left(x_{i+1}, y, t\right)\right)-f\left(u\left(x_{i}, y, t\right)\right)\right] \frac{y-y_{j+1 / 2}}{\Delta y} d y d t \\
& -\frac{1}{2 \Delta x \Delta y} \int_{t^{n}}^{t^{n+1}} \int_{x_{i}}^{x_{i+1}}\left[g\left(u\left(x, y_{j+1}, t\right)\right)+g\left(u\left(x, y_{j}, t\right)\right)\right] d x d t \\
& +\frac{1}{\Delta x \Delta y^{2}} \int_{t^{n}}^{t^{n+1}} \int_{x_{i}}^{x_{i+1}} \int_{y_{j}}^{y_{j+1}} g(u(x, y, t)) d x d y d t, \\
& \frac{1}{\Delta x \Delta y} \int_{I_{i+1 / 2, j+1 / 2}} u\left(x, y, t^{n+1}\right) \frac{x-x_{i+1 / 2}}{\Delta x} \frac{y-y_{j+1 / 2}}{\Delta y} d x d y \\
& =\frac{1}{\Delta x \Delta y} \int_{I_{i+1 / 2, j+1 / 2}} u\left(x, y, t^{n}\right) \frac{x-x_{i+1 / 2}}{\Delta x} \frac{y-y_{j+1 / 2}}{\Delta y} d x d y \\
& -\frac{1}{2 \Delta x \Delta y} \int_{t^{n}}^{t^{n+1}} \int_{y_{j}}^{y_{j+1}}\left[f\left(u\left(x_{i+1}, y, t\right)\right)+f\left(u\left(x_{i}, y, t\right)\right)\right] \frac{y-y_{j+1 / 2}}{\Delta y} d y d t \\
& +\frac{1}{\Delta x^{2} \Delta y} \int_{t^{n}}^{t^{n+1}} \int_{x_{i}}^{x_{i+1}} \int_{y_{j}}^{y_{j+1}} f(u(x, y, t)) \frac{y-y_{j+1 / 2}}{\Delta y} d x d y d t \\
& -\frac{1}{2 \Delta x \Delta y} \int_{t^{n}}^{t^{n+1}} \int_{x_{i}}^{x_{i+1}}\left[g\left(u\left(x, y_{j+1}, t\right)\right)+g\left(u\left(x, y_{j}, t\right)\right)\right] \frac{x-x_{i+1 / 2}}{\Delta x} d x d t \\
& +\frac{1}{\Delta x \Delta y^{2}} \int_{t^{n}}^{t^{n+1}} \int_{x_{i}}^{x_{i+1}} \int_{y_{j}}^{y_{j+1}} g(u(x, y, t)) \frac{x-x_{i+1 / 2}}{\Delta x} d x d y d t .
\end{aligned}
$$

Here $\Delta t=t^{n+1}-t^{n}$ is the time step.

Just as in the one-dimensional case, we will approximate some spatial integral terms using numerical quadrature, and apply in time the Lax-Wendroff methodology. The resulted schemes will be fifth-order accurate in space and third-order in time, yet the ideas can be extended to general cases. To get the proposed method, for all the spatial integral terms involving $f(u)$ and $g(u)$ in (2.28)-(2.31), the one-dimensional fourpoint Gauss-Lobatto quadrature, or its tensor-version in two dimensions, will be used; In addition, if we assume the solution at $t^{n}$ is piecewise constant with respect to the primal mesh, and the time step $\Delta t$ satisfies the CFL restriction $\Delta t \leq \frac{C_{c f l}}{\left(\max \left|f^{\prime}(u)\right| / \Delta x+\max \left|g^{\prime}(u)\right| / \Delta y\right)}$ with some constant $C_{c f l}$, one can then expect $u\left(x_{*}, y_{*}, t\right)$ is smooth with respect to $t \in\left(t^{n}, t^{n+1}\right)$ at a quadrature point $\left(x_{*}, y_{*}\right) \in \mathcal{G}$. Here $\mathcal{G}$ denotes the collection of Gauss-Lobatto quadrature points with respect to the dual mesh. In this case, the flux functions 
$f\left(u\left(x_{*}, y_{*}, t\right)\right)$ and $g\left(u\left(x_{*}, y_{*}, t\right)\right)$ in $(2.28)-(2.31)$ can be approximated by a temporal Taylor expansion at $t^{n}$ as follows

$$
\begin{aligned}
& f\left(u\left(x_{*}, y_{*}, t\right)\right) \approx f\left(u\left(x_{*}, y_{*}, t^{n}\right)\right)+\left(t-t^{n}\right) f_{t}\left(u\left(x_{*}, y_{*}, t^{n}\right)\right)+\frac{\left(t-t^{n}\right)^{2}}{2} f_{t t}\left(u\left(x_{*}, y_{*}, t^{n}\right)\right), \\
& g\left(u\left(x_{*}, y_{*}, t\right)\right) \approx g\left(u\left(x_{*}, y_{*}, t^{n}\right)\right)+\left(t-t^{n}\right) g_{t}\left(u\left(x_{*}, y_{*}, t^{n}\right)\right)+\frac{\left(t-t^{n}\right)^{2}}{2} g_{t t}\left(u\left(x_{*}, y_{*}, t^{n}\right)\right) .
\end{aligned}
$$

Finally all time derivatives in (2.32) will be converted to spatial derivatives by repeatedly using the governing equations.

By combining (2.28)-(2.31) and the above-mentioned strategies in space and time, we are ready to present the proposed central scheme,

(b.1) Suppose at $t=t^{n}$, the approximations for all the zeroth-order and the first-order moments of the solution, denoted as $\bar{u}_{i j}^{n}, \bar{v}_{i j}^{n}, \bar{w}_{i j}^{n}, \overline{v w}_{i j}^{n}$, are available on the primal meshes, that is, $\forall i, j$,

$$
\begin{aligned}
& \bar{u}_{i j}^{n} \approx \frac{1}{\Delta x \Delta y} \int_{I_{i j}} u\left(x, y, t^{n}\right) d x d y, \quad \bar{v}_{i j}^{n} \approx \frac{1}{\Delta x \Delta y} \int_{I_{i j}} u\left(x, y, t^{n}\right) \frac{x-x_{i}}{\Delta x} d x d y, \\
& \bar{w}_{i j}^{n} \approx \frac{1}{\Delta x \Delta y} \int_{I_{i j}} u\left(x, y, t^{n}\right) \frac{y-y_{j}}{\Delta y} d x d y, \quad \overline{v w}_{i j}^{n} \approx \frac{1}{\Delta x \Delta y} \int_{I_{i j}} u\left(x, y, t^{n}\right) \frac{x-x_{i}}{\Delta x} \frac{y-y_{j}}{\Delta y} d x d y .
\end{aligned}
$$

Following a staggered central scheme strategy, we compute the zeroth-order and the first-order moments of the solution with respect to the dual mesh at $t=t^{n+1}$, denoted as $\bar{u}_{i+1 / 2, j+1 / 2}^{n+1}, \bar{v}_{i+1 / 2, j+1 / 2}^{n+1}$, $\bar{w}_{i+1 / 2, j+1 / 2}^{n+1}$ and $\overline{v w}_{i+1 / 2, j+1 / 2}^{n+1}$, as follows,

$$
\begin{aligned}
\bar{u}_{i+1 / 2, j+1 / 2}^{n+1}= & \bar{u}_{i+1 / 2, j+1 / 2}^{n}-\frac{\Delta t}{\Delta x} \sum_{s=1}^{4} \omega_{s}\left[F\left(u\left(x_{i+1}, \mathcal{G}_{s}^{y}, t^{n}\right)\right)-F\left(u\left(x_{i}, \mathcal{G}_{s}^{y}, t^{n}\right)\right)\right] \\
& -\frac{\Delta t}{\Delta y} \sum_{l=1}^{4} \omega_{l}\left[G\left(u\left(\mathcal{G}_{l}^{x}, y_{j+1}, t^{n}\right)\right)-G\left(u\left(\mathcal{G}_{l}^{x}, y_{j}, t^{n}\right)\right)\right] \\
\bar{v}_{i+1 / 2, j+1 / 2}^{n+1} & =\bar{v}_{i+1 / 2, j+1 / 2}^{n}-\frac{\Delta t}{2 \Delta x} \sum_{s=1}^{4} \omega_{s}\left[F\left(u\left(x_{i+1}, \mathcal{G}_{s}^{y}, t^{n}\right)\right)+F\left(u\left(x_{i}, \mathcal{G}_{s}^{y}, t^{n}\right)\right)\right] \\
& +\frac{\Delta t}{\Delta x} \sum_{l=1}^{4} \sum_{s=1}^{4} \omega_{l} \omega_{s} F\left(u\left(\mathcal{G}_{l}^{x}, \mathcal{G}_{s}^{y}, t^{n}\right)\right) \\
& -\frac{\Delta t}{\Delta y} \sum_{l=1}^{4} \omega_{l}\left[G\left(u\left(\mathcal{G}_{l}^{x}, y_{j+1}, t^{n}\right)\right)-G\left(u\left(\mathcal{G}_{l}^{x}, y_{j}, t^{n}\right)\right)\right] \frac{\mathcal{G}_{l}^{x}-x_{i+1 / 2}}{\Delta x} \\
\bar{w}_{i+1 / 2, j+1 / 2}^{n+1}= & \bar{w}_{i+1 / 2, j+1 / 2}^{n}-\frac{\Delta t}{\Delta x} \sum_{s=1}^{4} \omega_{s}\left[F\left(u\left(x_{i+1}, \mathcal{G}_{s}^{y}, t^{n}\right)\right)-F\left(u\left(x_{i}, \mathcal{G}_{s}^{y}, t^{n}\right)\right)\right] \frac{\mathcal{G}_{s}^{y}-y_{j+1 / 2}}{\Delta y} \\
- & \frac{\Delta t}{2 \Delta y} \sum_{l=1}^{4} \omega_{l}\left[G\left(u\left(\mathcal{G}_{l}^{x}, y_{j+1}, t^{n}\right)\right)+G\left(u\left(\mathcal{G}_{l}^{x}, y_{j}, t^{n}\right)\right)\right] \\
+ & \frac{\Delta t}{\Delta y} \sum_{l=1}^{4} \sum_{s=1}^{4} \omega_{l} \omega_{s} G\left(u\left(\mathcal{G}_{l}^{x}, \mathcal{G}_{s}^{y}, t^{n}\right)\right),
\end{aligned}
$$


and

$$
\begin{aligned}
\overline{v w}_{i+1 / 2, j+1 / 2}^{n+1} & =\overline{v w}_{i+1 / 2, j+1 / 2}^{n}-\frac{\Delta t}{2 \Delta x} \sum_{s=1}^{4} \omega_{s}\left[F\left(u\left(x_{i+1}, \mathcal{G}_{s}^{y}, t^{n}\right)\right)+F\left(u\left(x_{i}, \mathcal{G}_{s}^{y}, t^{n}\right)\right)\right] \frac{\mathcal{G}_{s}^{y}-y_{j+1 / 2}}{\Delta y} \\
& +\frac{\Delta t}{\Delta x} \sum_{l=1}^{4} \sum_{s=1}^{4} \omega_{l} \omega_{s} F\left(u\left(\mathcal{G}_{l}^{x}, \mathcal{G}_{s}^{y}, t^{n}\right)\right) \frac{\mathcal{G}_{s}^{y}-y_{j+1 / 2}}{\Delta y} \\
& -\frac{\Delta t}{2 \Delta y} \sum_{l=1}^{4} \omega_{l}\left[G\left(u\left(\mathcal{G}_{l}^{x}, y_{j+1}, t^{n}\right)\right)+G\left(u\left(\mathcal{G}_{l}^{x}, y_{j}, t^{n}\right)\right)\right] \frac{\mathcal{G}_{l}^{x}-x_{i+1 / 2}}{\Delta x} \\
& +\frac{\Delta t}{\Delta y} \sum_{l=1}^{4} \sum_{s=1}^{4} \omega_{l} \omega_{s} G\left(u\left(\mathcal{G}_{l}^{x}, \mathcal{G}_{s}^{y}, t^{n}\right)\right) \frac{\mathcal{G}_{l}^{x}-x_{i+1 / 2}}{\Delta x} .
\end{aligned}
$$

Here

$$
\begin{aligned}
& F(u)=f(u)+\frac{\Delta t}{2} f_{t}(u)+\frac{\Delta t^{2}}{6} f_{t t}(u)=f(u)+\frac{\Delta t}{2} f^{\prime}(u) u_{t}+\frac{\Delta t^{2}}{6}\left(f^{\prime \prime}(u)\left(u_{t}\right)^{2}+f^{\prime}(u) u_{t t}\right) \\
& G(u)=g(u)+\frac{\Delta t}{2} g_{t}(u)+\frac{\Delta t^{2}}{6} g_{t t}(u)=g(u)+\frac{\Delta t}{2} g^{\prime}(u) u_{t}+\frac{\Delta t^{2}}{6}\left(g^{\prime \prime}(u)\left(u_{t}\right)^{2}+g^{\prime}(u) u_{t t}\right)
\end{aligned}
$$

with all the time derivatives of $u$ converted to the spatial ones according to the following

$$
\begin{aligned}
u_{t} & =-f(u)_{x}-g(u)_{y}=-\left(f^{\prime}(u) u_{x}+g^{\prime}(u) u_{y}\right), \\
u_{x t} & =-\left(f^{\prime \prime}(u) u_{x}^{2}+f^{\prime}(u) u_{x x}+g^{\prime \prime}(u) u_{x} u_{y}+g^{\prime}(u) u_{x y}\right), \\
u_{y t} & =-\left(f^{\prime \prime}(u) u_{y} u_{x}+f^{\prime}(u) u_{x y}+g^{\prime \prime}(u) u_{y}^{2}+g^{\prime}(u) u_{y y}\right), \\
u_{t t} & =-\left(f^{\prime}(u) u_{t}\right)_{x}-\left(g^{\prime}(u) u_{t}\right)_{y}=-\left(f^{\prime \prime}(u) u_{x} u_{t}+f^{\prime}(u) u_{x t}+g^{\prime \prime}(u) u_{y} u_{t}+g^{\prime}(u) u_{y t}\right) .
\end{aligned}
$$

Again, the notation $u$ is no longer the exact solution, and its relevant values will be reconstructed. $\mathcal{G}_{s}^{y}$, $s=1,2,3,4$ are the counterpart of (2.6) in $y$-direction.

(b.2) With $\bar{u}_{i+1 / 2, j+1 / 2}^{n+1}, \bar{v}_{i+1 / 2, j+1 / 2}^{n+1}, \bar{w}_{i+1 / 2, j+1 / 2}^{n+1}$ and $\bar{v}_{i+1 / 2, j+1 / 2}^{n+1}$ being available for any $i, j$, we further compute all the zeroth-order and the first-order moments of the solution with respect to the primal mesh at $t=t^{n+2}$, denoted as $\bar{u}_{i j}^{n+2}, \bar{v}_{i j}^{n+2}, \bar{w}_{i j}^{n+2}$ and $\overline{v w}_{i j}^{n+2}$. The formulations are omitted here for simplicity.

(b.3) Set $n$ to be $n+2$, and go to (b.1).

Note that the mesh switches back after two time steps.

To finalize the proposed scheme, one needs to reconstruct certain quantities in (b.1) and (b.2). With similarity, we will only focus on (b.1). In this step, to obtain the moments $\bar{u}_{i+1 / 2, j+1 / 2}^{n+1}, \bar{v}_{i+1 / 2, j+1 / 2}^{n+1}$, $\bar{w}_{i+1 / 2, j+1 / 2}^{n+1}$ and $\overline{v w}_{i+1 / 2, j+1 / 2}^{n+1}$ on the dual mesh at the next time $t^{n+1}$ based on (2.34)-(2.37), one will need to reconstruct at the current time $t^{n}, \forall i, j$,

(1) the moments $\bar{u}_{i+1 / 2, j+1 / 2}^{n}, \bar{v}_{i+1 / 2, j+1 / 2}^{n}, \bar{w}_{i+1 / 2, j+1 / 2}^{n}$ and $\overline{v w}_{i+1 / 2, j+1 / 2}^{n}$ on the dual mesh;

(2) the point value of $q\left(x_{*}, y_{*}, t^{n}\right)$, where $q=u, u_{x}, u_{y}, u_{x x}, u_{x y}$ or $u_{y y}$, and they are the functions $F$ and $G$ in (2.38) actually depend on. Here $\left(x_{*}, y_{*}\right) \in \mathcal{G}$ is some interior point with respect to the primal mesh, coming from the Gauss-Lobatto quadrature nodes on the dual mesh. 
In the same spirit as in one dimension, to obtain the cell average $\bar{u}_{i+1 / 2, j+1 / 2}^{n}, \forall i, j$, one would want to get the following four quarter-cell averages

$$
\begin{array}{ll}
\frac{1}{\Delta x \Delta y} \int_{x_{i}}^{x_{i+1 / 2}} \int_{y_{j}}^{y_{j+1 / 2}} u\left(x, y, t^{n}\right) d x d y, & \frac{1}{\Delta x \Delta y} \int_{x_{i+1 / 2}}^{x_{i+1}} \int_{y_{j}}^{y_{j+1 / 2}} u\left(x, y, t^{n}\right) d x d y \\
\frac{1}{\Delta x \Delta y} \int_{x_{i}}^{x_{i+1 / 2}} \int_{y_{j+1 / 2}}^{y_{j+1}} u\left(x, y, t^{n}\right) d x d y, & \frac{1}{\Delta x \Delta y} \int_{x_{i+1 / 2}}^{x_{i+1}} \int_{y_{j+1 / 2}}^{y_{j+1}} u\left(x, y, t^{n}\right) d x d y .
\end{array}
$$

This similarly goes to all the first-order moments.

To reconstruct the quarter-cell moments and point values mentioned above, we propose a dimension-bydimension procedure and apply the one-dimensional fifth-order accurate HWENO reconstruction in Section 2.1 multiple times. The dimension-by-dimension reconstruction greatly improves the ease of the implementation in high dimensions. Such treatment is possible in our proposed work mainly due to the inclusion of the mixed moment $\overline{v w}_{i j}^{n}$ which was introduced in [38]. Without such term, accurate and stable reconstructions can still be formulated, yet they would need to be implemented in a truly two dimensional fashion. In the remaining of this section, we will describe the reconstruction in details through steps 1-5, where the reconstruction is based on the moments $\left\{\bar{u}_{i j}^{n}, \bar{v}_{i j}^{n}, \bar{w}_{i j}^{n}, \overline{v w}_{i j}^{n}\right\}_{i j}$ on the primal mesh at $t=t^{n}$, and is accomplished by applying the one-dimensional fifth-order reconstruction in Section 2.1. The superscript $n$ will be omitted, together with the dependence on the time $t$ of all the involved functions.

Step 1. Along $x$ direction, based on $\left\{\bar{u}_{i j}, \bar{v}_{i j}\right\}_{i j}$, we reconstruct

$$
\left\{\begin{array}{l}
\frac{1}{\Delta x \Delta y} \int_{x_{i-1 / 2}}^{x_{i}} \int_{y_{j-1 / 2}}^{y_{j+1 / 2}} u(x, y) d x d y, \quad \frac{1}{\Delta x \Delta y} \int_{x_{i}}^{x_{i+1 / 2}} \int_{y_{j-1 / 2}}^{y_{j+1 / 2}} u(x, y) d x d y \\
\frac{1}{\Delta x \Delta y} \int_{x_{i-1 / 2}}^{x_{i}} \int_{y_{j-1 / 2}}^{y_{j+1 / 2}} u(x, y) \frac{x-x_{i-1 / 2}}{\Delta x} d x d y, \quad \frac{1}{\Delta x \Delta y} \int_{x_{i}}^{x_{i+1 / 2}} \int_{y_{j-1 / 2}}^{y_{j+1 / 2}} u(x, y) \frac{x-x_{i+1 / 2}}{\Delta x} d x d y \\
\frac{1}{\Delta y} \int_{y_{j-1 / 2}}^{y_{j+1 / 2}} p\left(x_{*}, y\right) d y, \quad p=u, u_{x}, u_{x x}, \quad x_{*} \in \mathcal{G}^{x, i}
\end{array}\right.
$$

Step 2. Along $x$ direction, based on $\left\{\bar{w}_{i j}, \overline{v w}_{i j}\right\}_{i j}$, we reconstruct

$$
\left\{\begin{array}{l}
\frac{1}{\Delta x \Delta y} \int_{x_{i-1 / 2}}^{x_{i}} \int_{y_{j-1 / 2}}^{y_{j+1 / 2}} u(x, y) \frac{y-y_{j}}{\Delta y} d x d y, \quad \frac{1}{\Delta x \Delta y} \int_{x_{i}}^{x_{i+1 / 2}} \int_{y_{j-1 / 2}}^{y_{j+1 / 2}} u(x, y) \frac{y-y_{j}}{\Delta y} d x d y \\
\frac{1}{\Delta x \Delta y} \int_{x_{i-1 / 2}}^{x_{i}} \int_{y_{j-1 / 2}}^{y_{j+1 / 2}} u(x, y) \frac{x-x_{i-1 / 2}}{\Delta x} \frac{y-y_{j}}{\Delta y} d x d y \\
\frac{1}{\Delta x \Delta y} \int_{x_{i}}^{x_{i+1 / 2}} \int_{y_{j-1 / 2}}^{y_{j+1 / 2}} u(x, y) \frac{x-x_{i+1 / 2}}{\Delta x} \frac{y-y_{j}}{\Delta y} d x d y \\
\frac{1}{\Delta y} \int_{y_{j-1 / 2}}^{y_{j+1 / 2}} p\left(x_{*}, y\right) \frac{y-y_{j}}{\Delta y} d y, \quad p=u, u_{x}, u_{x x}, \quad x_{*} \in \mathcal{G}^{x, i} .
\end{array}\right.
$$

Step 3. Along $y$ direction, based on

$$
\frac{1}{\Delta x \Delta y} \int_{x_{i-1 / 2}}^{x_{i}} \int_{y_{j-1 / 2}}^{y_{j+1 / 2}} u(x, y) d x d y, \quad \frac{1}{\Delta x \Delta y} \int_{x_{i-1 / 2}}^{x_{i}} \int_{y_{j-1 / 2}}^{y_{j+1 / 2}} u(x, y) \frac{y-y_{j}}{\Delta y} d x d y
$$


with all $i, j$, we reconstruct

$$
\left\{\begin{array}{l}
\frac{1}{\Delta x \Delta y} \int_{x_{i-1 / 2}}^{x_{i}} \int_{y_{j-1 / 2}}^{y_{j}} u(x, y) d x d y, \quad \frac{1}{\Delta x \Delta y} \int_{x_{i-1 / 2}}^{x_{i}} \int_{y_{j}}^{y_{j+1 / 2}} u(x, y) d x d y, \\
\frac{1}{\Delta x \Delta y} \int_{x_{i-1 / 2}}^{x_{i}} \int_{y_{j-1 / 2}}^{y_{j}} u(x, y) \frac{y-y_{j-1 / 2}}{\Delta y} d x d y, \frac{1}{\Delta x \Delta y} \int_{x_{i-1 / 2}}^{x_{i}} \int_{y_{j}}^{y_{j+1 / 2}} u(x, y) \frac{y-y_{j+1 / 2}}{\Delta y} d x d y .
\end{array}\right.
$$

Similarly, based on

$$
\frac{1}{\Delta x \Delta y} \int_{x_{i}}^{x_{i+1 / 2}} \int_{y_{j-1 / 2}}^{y_{j+1 / 2}} u(x, y) d x d y, \quad \frac{1}{\Delta x \Delta y} \int_{x_{i}}^{x_{i+1 / 2}} \int_{y_{j-1 / 2}}^{y_{j+1 / 2}} u(x, y) \frac{y-y_{j}}{\Delta y} d x d y
$$

with all $i, j$, we reconstruct

$$
\left\{\begin{array}{l}
\frac{1}{\Delta x \Delta y} \int_{x_{i}}^{x_{i+1 / 2}} \int_{y_{j-1 / 2}}^{y_{j}} u(x, y) d x d y, \frac{1}{\Delta x \Delta y} \int_{x_{i}}^{x_{i+1 / 2}} \int_{y_{j}}^{y_{j+1 / 2}} u(x, y) d x d y \\
\frac{1}{\Delta x \Delta y} \int_{x_{i}}^{x_{i+1 / 2}} \int_{y_{j-1 / 2}}^{y_{j}} u(x, y) \frac{y-y_{j-1 / 2}}{\Delta y} d x d y, \frac{1}{\Delta x \Delta y} \int_{x_{i}}^{x_{i+1 / 2}} \int_{y_{j}}^{y_{j+1 / 2}} u(x, y) \frac{y-y_{j+1 / 2}}{\Delta y} d x d y .
\end{array}\right.
$$

Step 4. Along $y$ direction, based on

$$
\frac{1}{\Delta x \Delta y} \int_{x_{i-1 / 2}}^{x_{i}} \int_{y_{j-1 / 2}}^{y_{j+1 / 2}} u(x, y) \frac{x-x_{i-1 / 2}}{\Delta x} d x d y, \quad \frac{1}{\Delta x \Delta y} \int_{x_{i-1 / 2}}^{x_{i}} \int_{y_{j-1 / 2}}^{y_{j+1 / 2}} u(x, y) \frac{x-x_{i-1 / 2}}{\Delta x} \frac{y-y_{j}}{\Delta y} d x d y
$$

with all $i, j$, we reconstruct

$$
\left\{\begin{array}{l}
\frac{1}{\Delta x \Delta y} \int_{x_{i-1 / 2}}^{x_{i}} \int_{y_{j-1 / 2}}^{y_{j}} u(x, y) \frac{x-x_{i-1 / 2}}{\Delta x} d x d y, \quad \frac{1}{\Delta x \Delta y} \int_{x_{i-1 / 2}}^{x_{i}} \int_{y_{j}}^{y_{j+1 / 2}} u(x, y) \frac{x-x_{i-1 / 2}}{\Delta x} d x d y \\
\frac{1}{\Delta x \Delta y} \int_{x_{i-1 / 2}}^{x_{i}} \int_{y_{j-1 / 2}}^{y_{j}} u(x, y) \frac{x-x_{i-1 / 2}}{\Delta x} \frac{y-y_{j-1 / 2}}{\Delta y} d x d y \\
\frac{1}{\Delta x \Delta y} \int_{x_{i-1 / 2}}^{x_{i}} \int_{y_{j}}^{y_{j+1 / 2}} u(x, y) \frac{x-x_{i-1 / 2}}{\Delta x} \frac{y-y_{j+1 / 2}}{\Delta y} d x d y .
\end{array}\right.
$$

Similarly, based on

$$
\frac{1}{\Delta x \Delta y} \int_{x_{i}}^{x_{i+1 / 2}} \int_{y_{j-1 / 2}}^{y_{j+1 / 2}} u(x, y) \frac{x-x_{i+1 / 2}}{\Delta x} d x d y, \quad \frac{1}{\Delta x \Delta y} \int_{x_{i}}^{x_{i+1 / 2}} \int_{y_{j-1 / 2}}^{y_{j+1 / 2}} u(x, y) \frac{x-x_{i+1 / 2}}{\Delta x} \frac{y-y_{j}}{\Delta y} d x d y
$$

with all $i, j$, we reconstruct

$$
\left\{\begin{array}{l}
\frac{1}{\Delta x \Delta y} \int_{x_{i}}^{x_{i+1 / 2}} \int_{y_{j-1 / 2}}^{y_{j}} u(x, y) \frac{x-x_{i+1 / 2}}{\Delta x} d x d y, \quad \frac{1}{\Delta x \Delta y} \int_{x_{i}}^{x_{i+1 / 2}} \int_{y_{j}}^{y_{j+1 / 2}} u(x, y) \frac{x-x_{i+1 / 2}}{\Delta x} d x d y \\
\frac{1}{\Delta x \Delta y} \int_{x_{i}}^{x_{i+1 / 2}} \int_{y_{j-1 / 2}}^{y_{j}} u(x, y) \frac{x-x_{i+1 / 2}}{\Delta x} \frac{y-y_{j-1 / 2}}{\Delta y} d x d y \\
\frac{1}{\Delta x \Delta y} \int_{x_{i}}^{x_{i+1 / 2}} \int_{y_{j}}^{y_{j+1 / 2}} u(x, y) \frac{x-x_{i+1 / 2}}{\Delta x} \frac{y-y_{j+1 / 2}}{\Delta y} d x d y .
\end{array}\right.
$$

Step 5. Along $y$ direction, based on

$$
\frac{1}{\Delta y} \int_{y_{j-1 / 2}}^{y_{j+1 / 2}} p\left(x_{*}, y\right) d y, \quad \frac{1}{\Delta y} \int_{y_{j-1 / 2}}^{y_{j+1 / 2}} p\left(x_{*}, y\right) \frac{y-y_{j}}{\Delta y} d y, \quad p=u, u_{x}, u_{x x}, \quad x_{*} \in \mathcal{G}^{x, i}
$$

with all $i, j$, we reconstruct

$$
q\left(x_{*}, y_{*}\right), \quad q=u, u_{x}, u_{y}, u_{x x}, u_{x y}, u_{y y}, \quad\left(x_{*}, y_{*}\right) \in \mathcal{G} .
$$


Remark 4. Similar as in the one-dimensional case (see also Remark 1), with the current central scheme framework, there is no need to use numerical fluxes for $F$ and $G$ as in upwind type schemes.

Finally in this section, we briefly discuss the system case. Just as in one dimension, to reduce the spurious oscillations and enhance the numerical stability, meanwhile to maintain good computational efficiency, we apply the local characteristic decomposition dimension-by-dimension during the reconstructions of the quarter-cell moments for $u$ based on $\left\{\bar{u}_{i j}, \bar{v}_{i j}, \bar{w}_{i j}, \overline{v w}_{i j}\right\}_{i j}$. The reconstructions of the point values are still implemented through a componentwise procedure. Again to assist with the derivation of the proposed methods, MATLAB Symbolic Math Toolbox is used.

\section{Central Hermite WENO schemes with natural continuous ex- tension of Runge-Kutta time discretization}

Alternative to the Lax-Wendroff strategy in Section 2, we apply in this section the natural continuous extension of Runge-Kutta (NCE-RK) time discretizations, to combine with a central HWENO spatial discretizations in the framework of staggered meshes. The use of NCE-RK methods permits one to compute accurate approximations for the intermediate value of a solution to an ODE based on standard RK methods with slight increase of the computational cost. Note that a standard RK method in general only provides accurate approximations for the solution at discrete time $t^{n}$ for any $n$.

Below we will describe a fourth-order NCE-RK method which is used in this paper. For more details about such time discretizations, one can refer to [4,37]. Consider a scalar or a system of ODE problem

$$
\left\{\begin{array}{l}
y^{\prime}(t)=H(t, y(t)) \\
y\left(t_{0}\right)=y_{0}
\end{array}\right.
$$

and suppose $y^{n}$ is a given approximation to $y\left(t^{n}\right)$. One can then approximate $y\left(t^{n+1}\right)$ at $t^{n+1}=t^{n}+\Delta t$ by $y^{n+1}$ with a standard four-stage fourth-order RK scheme as follows.

$$
y^{n+1}=y^{n}+\Delta t \sum_{i=1}^{4} b_{i} K^{(i)},
$$

where $K^{(i)}$ is an RK flux determined by

$$
K^{(i)}=H\left(t^{n}+c_{i} \Delta t, Y^{(i)}\right) \quad \text { with } Y^{(i)}=y^{n}+c_{i} \Delta t K^{(i-1)}, \quad i=1,2,3,4,
$$

and $K^{(0)}=0$. In addition, $b_{1}=b_{4}=\frac{1}{6}, b_{2}=b_{3}=\frac{1}{3}$, and $c_{1}=0, c_{2}=c_{3}=\frac{1}{2}, c_{4}=1$.

A natural continuous extension of the RK scheme (3.2)-(3.3) further provides an approximation for $y(t)$ (and also its derivatives) with the same accuracy when $t \in\left[t^{n}, t^{n+1}\right]$. This approximation is given specifically by

$$
\left.s(t)\right|_{t=t^{n}+\theta \Delta t}:=y^{n}+\Delta t \sum_{i=1}^{4} B_{i}(\theta) K^{(i)}, \quad 0 \leq \theta \leq 1,
$$

where

$$
\begin{aligned}
& B_{1}(\theta)=2\left(1-4 b_{1}\right) \theta^{3}+3\left(3 b_{1}-1\right) \theta^{2}+\theta, \\
& B_{i}(\theta)=4\left(3 c_{i}-2\right) b_{i} \theta^{3}+3\left(3-4 c_{i}\right) b_{i} \theta^{2}, \quad i=2,3,4 .
\end{aligned}
$$

$s(t)$ not only satisfies $s\left(t^{n}\right)=y^{n}, s\left(t^{n+1}\right)=y^{n+1}$, but also has the following approximation properties,

$$
\max _{t^{n} \leq t \leq t^{n}+\Delta t}\left|y^{(l)}(t)-s^{(l)}(t)\right|=O\left(\Delta t^{4-l}\right), \quad 0 \leq l \leq 4 .
$$




\subsection{One-dimensional case}

We use the same notation for the staggered meshes and the moments of a function as in Section 2.1. Though the discussion below focuses on one time step from $t=t^{n}$ to $t=t^{n+1}$, one would want to keep in mind that the overall algorithm is still based on staggered meshes, and it switches back and forth between the primal and dual meshes.

To derive our scheme, we follow the initial presentation in Section 2.1 all the way till equation (2.5), with one line integral of $f(u)$ in $x$-variable approximated by numerical quadrature in (2.5). Using a similar argument in Section 2.1, under suitable assumption on the time step $\Delta t, f\left(u\left(x_{*}, t\right)\right)$ can be regarded to be smooth for $t \in\left(t^{n}, t^{n+1}\right)$ when $x_{*} \in \mathcal{G}^{x}$. Now to discretize in time, we will adopt NCE-RK approach here instead of the Lax-Wendroff methodology presented in Section 2.1. To this end, we apply a three-point Gaussian quadrature formula and approximate the temporal integral terms in (2.3) and (2.5) according to the following

$$
\int_{t^{n}}^{t^{n+1}} f\left(u\left(x_{*}, t\right)\right) d t \approx \Delta t \sum_{m=1}^{3} \alpha_{m} f\left(u\left(x_{*}, t^{n}+\Delta t \theta_{m}\right)\right) .
$$

Here $\alpha_{1}=\alpha_{3}=\frac{5}{18}, \alpha_{2}=\frac{4}{9}$ are the quadrature weights, and $\theta_{1}=\frac{1}{2}-\frac{\sqrt{15}}{10}, \theta_{2}=\frac{1}{2}, \theta_{3}=\frac{1}{2}+\frac{\sqrt{15}}{10}$ are the quadrature points.

With this new treatment in time, we are ready to present the proposed scheme from $t^{n}$ to $t^{n+1}$, a counterpart of step (a.1) in Section 2.1.

(a'.1) Suppose at $t=t^{n}$, the approximations for the first two moments of the solution, denoted as $\left\{\bar{u}_{i}^{n}\right\}$ and $\left\{\bar{v}_{i}^{n}\right\}$, are available on the primal mesh $\forall i$, see (2.12). Following a staggered central scheme strategy, we compute the first two moments of the solution with respect to the dual mesh at $t=t^{n+1}$, denoted as $\bar{u}_{i+1 / 2}^{n+1}$ and $\bar{v}_{i+1 / 2}^{n+1}$, as follows,

$$
\begin{aligned}
& \bar{u}_{i+1 / 2}^{n+1}=\bar{u}_{i+1 / 2}^{n}-\left.\frac{\Delta t}{\Delta x} \sum_{m=1}^{3} \alpha_{m}\left[f\left(u\left(x_{i+1}, t\right)\right)-f\left(u\left(x_{i}, t\right)\right)\right]\right|_{t=t^{n}+\Delta t \theta_{m}}, \\
& \bar{v}_{i+1 / 2}^{n+1}=\bar{v}_{i+1 / 2}^{n}-\left.\frac{\Delta t}{\Delta x} \sum_{m=1}^{3} \alpha_{m}\left[\frac{1}{2} f\left(u\left(x_{i+1}, t\right)\right)+\frac{1}{2} f\left(u\left(x_{i}, t\right)\right)-\sum_{l=1}^{4} \omega_{l} f\left(u\left(\mathcal{G}_{l}^{x}, t\right)\right)\right]\right|_{t=t^{n}+\Delta t \theta_{m}} .
\end{aligned}
$$

Again, the notation $u$ is no longer for the exact solution, but to be reconstructed.

Based on (3.5)-(3.6), to compute the moments at $t=t^{n+1}$, namely, $\left\{\bar{u}_{i+1 / 2}^{n+1}\right\}_{i}$ and $\left\{\bar{v}_{i+1 / 2}^{n+1}\right\}_{i}$, with respect to the dual mesh, one would need to accurately approximate the following quantities

$$
\bar{u}_{i+1 / 2}^{n}, \bar{v}_{i+1 / 2}^{n}, \forall i, \quad u\left(x_{*}, t^{n}+\Delta t \theta_{m}\right), m=1,2,3, x_{*} \in \mathcal{G}^{x} .
$$

Recall at $t^{n},\left\{\bar{u}_{i}^{n}, \bar{v}_{i}^{n}\right\}_{i}$ are available. To reconstruct the staggered moments $\left\{\bar{u}_{i+1 / 2}^{n}\right\}_{i}$ and $\left\{\bar{v}_{i+1 / 2}^{n}\right\}_{i}$ associated with the dual mesh in (3.7), one can apply the fifth-order HWENO procedure described in Section 2.1 .

To approximate the point values $u\left(x_{*}, t^{n}+\Delta t \theta_{m}\right), m=1,2,3, x_{*} \in \mathcal{G}^{x}$ in (3.7), we will apply the fourth-order NCE-RK method to an auxiliary ODE problem (3.1) starting from $t=t^{n}$, where $y(t)$ in (3.1) is the collection of $u\left(x_{*}, t\right), \forall x_{*} \in \mathcal{G}^{x}$, and $-H(t, y)$ in (3.1) is a vector-valued functional which reconstructs $f_{x}\left(u\left(x_{*}, t\right)\right), \forall x_{*} \in \mathcal{G}^{x}$, based on $y(t)$, and is denoted as $\mathcal{R}(y(t))$. That is, $H(t, y(t))=-\mathcal{R}(y(t))$. To complete 
the description of this ODE problem, hence the strategy to approximate the point values $u\left(x_{*}, t^{n}+\Delta t \theta_{m}\right)$ in (3.7), two more ingredients are needed. One is the initial value $f\left(u\left(x_{*}, t^{n}\right)\right)$, namely, $u\left(x_{*}, t^{n}\right), \forall x_{*} \in \mathcal{G}^{x}$, and it can be reconstructed with the fifth-order HWENO procedure based on the given moments $\left\{\bar{u}_{i}^{n}, \bar{v}_{i}^{n}\right\}_{i}$ as discussed in step 3 of Section 2.1. The other ingredient is the functional $\mathcal{R}(y(t))$, which will be specified in the remaining of this subsection.

To reconstruct $f_{x}\left(u\left(x_{*}, t\right)\right), \forall x_{*} \in \mathcal{G}^{x}$ based on $u\left(x_{*}, t\right), \forall x_{*} \in \mathcal{G}^{x}$, we propose a fifth-order accurate WENO reconstruction. Though a HWENO reconstruction can also be formulated, the WENO reconstruction proposed here has much better cost efficiency without increasing the stencil. For any given $i$, consider all the Gauss-Lobatto quadrature points in the cell $I_{i-1 / 2}$, relabeled as $p_{1}, \cdots, p_{4}$, namely

$$
p_{1}=x_{i-1}, \quad p_{2}=x_{i-\frac{1}{2}-\frac{\sqrt{5}}{10}}, \quad p_{3}=x_{i-\frac{1}{2}+\frac{\sqrt{5}}{10}}, \quad p_{4}=x_{i} .
$$

With (mirror) symmetry, we only present the reconstruction of $f_{x}\left(u\left(x_{*}, t\right)\right)$, with $x_{*}=p_{3}$ and $p_{4}$. For the reconstruction, we will also use the neighboring Gauss-Lobatto points from $I_{i+1 / 2}$, given as

$$
p_{4}=x_{i}, \quad p_{5}=x_{i+\frac{1}{2}-\frac{\sqrt{5}}{10}}, \quad p_{6}=x_{i+\frac{1}{2}+\frac{\sqrt{5}}{10}}, \quad p_{7}=x_{i+1} .
$$

Note that the points $p_{1}, \cdots, p_{7}$ are interior points with respect to the primal mesh, see Figure 1 . In the

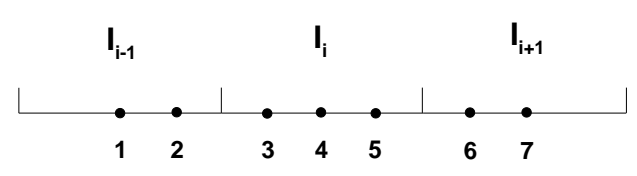

Figure 1: One-dimensional Gauss-Lobatto points in the cells $\left\{I_{i-1 / 2}, I_{i+1 / 2}\right\}$.

next two steps, we will reconstruct $f_{x}\left(u\left(x_{*}, t\right)\right)$ with $x_{*}=p_{3}$ and $p_{4}$, respectively, based on $u\left(x_{*}, t\right)$, with $x_{*}=p_{1}, \cdots, p_{6}$. As some shorthand notation, we write $f\left(u\left(p_{s}, t\right)\right)=f_{s}$, and $f_{x}\left(u\left(p_{s}, t\right)\right)=f_{x}\left(p_{s}\right)$.

Step 1: A WENO reconstruction of $f_{x}\left(p_{3}\right)$ from the point values $f_{s}, s=1, \cdots, 5$.

We first introduce three "small" stencils $S_{l}=\left\{p_{l+1}, p_{l+2}, p_{l+3}\right\}, l=0,1,2$, one "large" stencil $\mathcal{T}=$ $\left\{S_{0}, S_{1}, S_{2}\right\}$, and reconstruct three quadratic polynomials $q_{0}(x), q_{1}(x), q_{2}(x)$ and one quartic polynomial $Q(x)$ such that

$$
q_{l}\left(p_{l+j}\right)=f_{l+j}, l=0,1,2, j=1,2,3, \quad Q\left(p_{j}\right)=f_{j}, j=1,2,3,4,5 .
$$

The point values of the first derivative of these polynomials at $p_{3}$ can be expressed in terms of the point values of the function $f$,

$$
\begin{aligned}
q_{0}^{\prime}\left(p_{3}\right) & =\frac{\sqrt{5}}{2 \Delta x}\left[2 f_{1}-(3+\sqrt{5}) f_{2}+(1+\sqrt{5}) f_{3}\right], \\
q_{1}^{\prime}\left(p_{3}\right) & =\frac{\sqrt{5}}{2 \Delta x}\left[(-3+\sqrt{5}) f_{2}+(1-\sqrt{5}) f_{3}+2 f_{4}\right], \\
q_{2}^{\prime}\left(p_{3}\right) & =\frac{5+\sqrt{5}}{4 \Delta x}\left[-3 f_{3}+4 f_{4}-f_{5}\right], \\
Q^{\prime}\left(p_{3}\right)=-\frac{1}{88 \Delta x}\left[(48-32 \sqrt{5}) f_{1}\right. & \left.+(-88+88 \sqrt{5}) f_{2}+(110+22 \sqrt{5}) f_{3}-(88+88 \sqrt{5}) f_{4}+(18+10 \sqrt{5}) f_{5}\right] .
\end{aligned}
$$


The linear weights $\gamma_{0}^{\prime}, \gamma_{1}^{\prime}, \gamma_{2}^{\prime}$ can be determined by requiring

$$
Q^{\prime}\left(p_{3}\right)=\sum_{j=0}^{2} \gamma_{j}^{\prime} q_{j}^{\prime}\left(p_{3}\right)
$$

hold when $\left\{f_{s}, s=1, \cdots, 5\right\}$ take arbitrary values, and this leads to

$$
\gamma_{0}^{\prime}=\frac{20-6 \sqrt{5}}{55}, \quad \gamma_{1}^{\prime}=\frac{30+2 \sqrt{5}}{55}, \quad \gamma_{2}^{\prime}=\frac{5+4 \sqrt{5}}{55} .
$$

We then compute the smoothness indicators $\beta_{j}, j=0,1,2$ as below

$$
\beta_{j}=\Delta x^{3} \int_{I_{i}}\left(\frac{\partial^{2}}{\partial x^{2}} q_{j}(x)\right)^{2} d x .
$$

Since we are reconstructing the first derivative of the function $f$, the smoothness indicator $\beta_{j}$ only depends on the second derivative of the quadratic polynomial $q_{j}(x)$. For the convenience of the actual implementation, the smoothness indicators $\left\{\beta_{j}\right\}_{j=0}^{2}$ can be further given in terms of the point values of function $f$,

$$
\left\{\begin{array}{l}
\beta_{0}=25\left[-2 f_{1}+(1+\sqrt{5}) f_{2}+(1-\sqrt{5}) f_{3}\right]^{2} \\
\beta_{1}=25\left[(-1+\sqrt{5}) f_{2}-(1+\sqrt{5}) f_{3}+2 f_{4}\right]^{2} \\
\beta_{2}=\frac{25}{4}(3+\sqrt{5})^{2}\left(f_{3}-2 f_{4}+f_{5}\right)^{2} .
\end{array}\right.
$$

With the smoothness indicators $\left\{\beta_{j}\right\}_{j}$ in (3.12) and the linear weights in (3.10), we can compute the nonlinear weights $\omega_{j}, j=0,1,2$,

$$
\omega_{j}=\frac{\bar{\omega}_{j}}{\sum_{k=0}^{2} \bar{\omega}_{k}}, \quad \text { where } \bar{\omega}_{k}=\frac{\gamma_{k}^{\prime}}{\left(\varepsilon+\beta_{k}\right)^{2}}, \quad k=0,1,2 .
$$

The positive parameter $\varepsilon$ is taken to be $10^{-6}$ in our actual simulation in this paper.

The final WENO reconstruction for $f_{x}\left(p_{3}\right)$ is now obtained as

$$
f_{x}\left(p_{3}\right) \approx \sum_{j=0}^{2} \omega_{j} q_{j}^{\prime}\left(p_{3}\right) .
$$

Step 2: A WENO reconstruction of $f_{x}\left(p_{4}\right)$ from the point values $f_{s}, s=2, \cdots, 6$.

We first introduce three "small" stencils $S_{l}=\left\{p_{l+2}, p_{l+3}, p_{l+4}\right\}, l=0,1,2$, one "large" stencil $\mathcal{T}=$ $\left\{S_{0}, S_{1}, S_{2}\right\}$, and reconstruct three quadratic polynomials $q_{0}(x), q_{1}(x), q_{2}(x)$ and one quartic polynomial $Q(x)$ such that

$$
q_{l}\left(p_{l+j}\right)=f_{l+j}, l=0,1,2, j=2,3,4, \quad Q\left(p_{j}\right)=f_{j}, j=2,3,4,5,6 .
$$

The point values of the first derivative of these polynomials at $p_{4}$ can be expressed in terms of the point values of the function $f$,

$$
\begin{aligned}
& q_{0}^{\prime}\left(p_{4}\right)=\frac{1}{2 \Delta x}\left[(-5+3 \sqrt{5}) f_{2}-(5+3 \sqrt{5}) f_{3}+10 f_{4}\right] \\
& q_{1}^{\prime}\left(p_{4}\right)=\frac{1}{4 \Delta x}\left[-(5+\sqrt{5}) f_{3}+(5+\sqrt{5}) f_{5}\right] \\
& q_{2}^{\prime}\left(p_{4}\right)=\frac{1}{2 \Delta x}\left[-10 f_{4}+(5+3 \sqrt{5}) f_{5}+(5-3 \sqrt{5}) f_{6}\right]
\end{aligned}
$$




$$
Q^{\prime}\left(p_{4}\right)=\frac{1}{2 \Delta x}\left[(-2+\sqrt{5}) f_{2}-(2+\sqrt{5}) f_{3}+(2+\sqrt{5}) f_{5}+(2-\sqrt{5}) f_{6}\right] .
$$

The linear weights $\gamma_{0}^{\prime}, \gamma_{1}^{\prime}, \gamma_{2}^{\prime}$ can be determined by requiring

$$
Q^{\prime}\left(p_{4}\right)=\sum_{j=0}^{2} \gamma_{j}^{\prime} q_{j}^{\prime}\left(p_{4}\right)
$$

hold when $\left\{f_{s}, s=2, \cdots, 6\right\}$ take arbitrary values, and this leads to

$$
\gamma_{0}^{\prime}=\frac{5-\sqrt{5}}{20}, \quad \gamma_{1}^{\prime}=\frac{5+\sqrt{5}}{10}, \quad \gamma_{2}^{\prime}=\frac{5-\sqrt{5}}{20} .
$$

We then compute the smoothness indicators $\beta_{j}, j=0,1,2$ as in (3.11) and they can be further given in terms of the point values of function $f$,

$$
\left\{\begin{array}{l}
\beta_{0}=25\left[(-1+\sqrt{5}) f_{2}-(1+\sqrt{5}) f_{3}+2 f_{4}\right]^{2} \\
\beta_{1}=\frac{25}{4}(3+\sqrt{5})^{2}\left(f_{3}-2 f_{4}+f_{5}\right)^{2} \\
\beta_{2}=25\left[-2 f_{4}+(1+\sqrt{5}) f_{5}+(1-\sqrt{5}) f_{6}\right]^{2}
\end{array}\right.
$$

With the smoothness indicators $\left\{\beta_{j}\right\}_{j}$ in (3.14) and the linear weights in (3.13), we can compute the nonlinear weights $\omega_{j}, j=0,1,2$,

$$
\omega_{j}=\frac{\bar{\omega}_{j}}{\sum_{k=0}^{2} \bar{\omega}_{k}}, \quad \text { where } \bar{\omega}_{k}=\frac{\gamma_{k}^{\prime}}{\left(\varepsilon+\beta_{k}\right)^{2}}, \quad k=0,1,2 .
$$

The final WENO reconstruction for $f_{x}\left(p_{4}\right)$ is now obtained as

$$
f_{x}\left(p_{4}\right) \approx \sum_{j=0}^{2} \omega_{j} q_{j}^{\prime}\left(p_{4}\right) .
$$

\subsection{Two-dimensional case}

In two dimensions, we use the same notation for the staggered meshes and the moments of a function as in Section 2.2. The discussion will just focus on the algorithm over one time step. To obtain the proposed method, we start with equations (2.28)-(2.31), and approximate those spatial integral terms involving $f(u)$ and $g(u)$ using one-dimensional four-point Gauss-Lobatto quadrature, or its tensor-version in two dimensions. In time, we approximate the temporal integrals according to (3.4). This will lead us to the proposed scheme from $t^{n}$ to $t^{n+1}$, a counterpart of (b.1) in Section 2.2.

(b'.1) Suppose at $t=t^{n}$, the approximations for all the zeroth-order and the first-order moments of the solution, denoted as $\bar{u}_{i j}^{n}, \bar{v}_{i j}^{n}, \bar{w}_{i j}^{n}, \overline{v w}_{i j}^{n}, \forall i, j$, are available on the primal meshes, see (2.33). Following a staggered central scheme strategy, we compute the zeroth-order and the first-order moments of the solution with respect to the dual mesh at $t=t^{n+1}$, denoted as $\bar{u}_{i+1 / 2, j+1 / 2}^{n+1}, \bar{v}_{i+1 / 2, j+1 / 2}^{n+1}, \bar{w}_{i+1 / 2, j+1 / 2}^{n+1}$ and $\overline{v w}_{i+1 / 2, j+1 / 2}^{n+1}$, as follows,

$$
\begin{aligned}
\bar{u}_{i+1 / 2, j+1 / 2}^{n+1} & =\bar{u}_{i+1 / 2, j+1 / 2}^{n}-\left.\frac{\Delta t}{\Delta x} \sum_{m=1}^{3} \sum_{s=1}^{4} \alpha_{m} \omega_{s}\left[f\left(u\left(x_{i+1}, \mathcal{G}_{s}^{y}, t\right)\right)-f\left(u\left(x_{i}, \mathcal{G}_{s}^{y}, t\right)\right)\right]\right|_{t=t^{n}+\Delta t \theta_{m}} \\
& -\left.\frac{\Delta t}{\Delta y} \sum_{m=1}^{3} \sum_{l=1}^{4} \alpha_{m} \omega_{l}\left[g\left(u\left(\mathcal{G}_{l}^{x}, y_{j+1}, t\right)\right)-g\left(u\left(\mathcal{G}_{l}^{x}, y_{j}, t\right)\right)\right]\right|_{t=t^{n}+\Delta t \theta_{m}},
\end{aligned}
$$




$$
\begin{aligned}
\bar{v}_{i+1 / 2, j+1 / 2}^{n+1}= & \bar{v}_{i+1 / 2, j+1 / 2}^{n}-\left.\frac{\Delta t}{2 \Delta x} \sum_{m=1}^{3} \sum_{s=1}^{4} \alpha_{m} \omega_{s}\left[f\left(u\left(x_{i+1}, \mathcal{G}_{s}^{y}, t\right)\right)+f\left(u\left(x_{i}, \mathcal{G}_{s}^{y}, t\right)\right)\right]\right|_{t=t^{n}+\Delta t \theta_{m}} \\
& +\frac{\Delta t}{\Delta x} \sum_{m=1}^{3} \sum_{l=1}^{4} \sum_{s=1}^{4} \alpha_{m} \omega_{l} \omega_{s} f\left(u\left(\mathcal{G}_{l}^{x}, \mathcal{G}_{s}^{y}, t^{n}+\Delta t \theta_{m}\right)\right) \\
& -\left.\frac{\Delta t}{\Delta y} \sum_{m=1}^{3} \sum_{l=1}^{4} \alpha_{m} \omega_{l}\left[g\left(u\left(\mathcal{G}_{l}^{x}, y_{j+1}, t\right)\right)-g\left(u\left(\mathcal{G}_{l}^{x}, y_{j}, t\right)\right)\right]\right|_{t=t^{n}+\Delta t \theta_{m}} \frac{\mathcal{G}_{l}^{x}-x_{i+1 / 2}}{\Delta x} \\
\bar{w}_{i+1 / 2, j+1 / 2}^{n+1}= & \bar{w}_{i+1 / 2, j+1 / 2}^{n}-\left.\frac{\Delta t}{\Delta x} \sum_{m=1}^{3} \sum_{s=1}^{4} \alpha_{m} \omega_{s}\left[f\left(u\left(x_{i+1}, \mathcal{G}_{s}^{y}, t\right)\right)-f\left(u\left(x_{i}, \mathcal{G}_{s}^{y}, t\right)\right)\right]\right|_{t=t^{n}+\Delta t \theta_{m}} \frac{\mathcal{G}_{s}^{y}-y_{j+1 / 2}}{\Delta y} \\
- & \left.\frac{\Delta t}{2 \Delta y} \sum_{m=1}^{3} \sum_{l=1}^{4} \alpha_{m} \omega_{l}\left[g\left(u\left(\mathcal{G}_{l}^{x}, y_{j+1}, t\right)\right)+g\left(u\left(\mathcal{G}_{l}^{x}, y_{j}, t\right)\right)\right]\right|_{t=t^{n}+\Delta t \theta_{m}} \\
+ & \frac{\Delta t}{\Delta y} \sum_{m=1}^{3} \sum_{l=1}^{4} \sum_{s=1}^{4} \alpha_{m} \omega_{l} \omega_{s} g\left(u\left(\mathcal{G}_{l}^{x}, \mathcal{G}_{s}^{y}, t^{n}+\Delta t \theta_{m}\right)\right),
\end{aligned}
$$

and

$$
\begin{aligned}
\overline{v w}_{i+1 / 2, j+1 / 2}^{n+1} & =\overline{v w}_{i+1 / 2, j+1 / 2}^{n}-\left.\frac{\Delta t}{2 \Delta x} \sum_{m=1}^{3} \sum_{s=1}^{4} \alpha_{m} \omega_{s}\left[f\left(u\left(x_{i+1}, \mathcal{G}_{s}^{y}, t\right)\right)+f\left(u\left(x_{i}, \mathcal{G}_{s}^{y}, t\right)\right)\right]\right|_{t=t^{n}+\Delta t \theta_{m}} \frac{\mathcal{G}_{s}^{y}-y_{j+1 / 2}}{\Delta y} \\
& +\frac{\Delta t}{\Delta x} \sum_{m=1}^{3} \sum_{l=1}^{4} \sum_{s=1}^{4} \alpha_{m} \omega_{l} \omega_{s} f\left(u\left(\mathcal{G}_{l}^{x}, \mathcal{G}_{s}^{y}, t^{n}+\Delta t \theta_{m}\right)\right) \frac{\mathcal{G}_{s}^{y}-y_{j+1 / 2}}{\Delta y} \\
& -\left.\frac{\Delta t}{2 \Delta y} \sum_{m=1}^{3} \sum_{l=1}^{4} \alpha_{m} \omega_{l}\left[g\left(u\left(\mathcal{G}_{l}^{x}, y_{j+1}, t\right)\right)+g\left(u\left(\mathcal{G}_{l}^{x}, y_{j}, t\right)\right)\right]\right|_{t=t^{n}+\Delta t \theta_{m}} \frac{\mathcal{G}_{l}^{x}-x_{i+1 / 2}}{\Delta x} \\
& +\frac{\Delta t}{\Delta y} \sum_{m=1}^{3} \sum_{l=1}^{4} \sum_{s=1}^{4} \alpha_{m} \omega_{l} \omega_{s} g\left(u\left(\mathcal{G}_{l}^{x}, \mathcal{G}_{s}^{y}, t^{n}+\Delta t \theta_{m}\right)\right) \frac{\mathcal{G}_{l}^{x}-x_{i+1 / 2}}{\Delta x}
\end{aligned}
$$

Based on (3.15)-(3.18), to compute the moments at $t=t^{n+1}$, namely

$$
\left\{\bar{u}_{i+1 / 2, j+1 / 2}^{n+1}, \bar{v}_{i+1 / 2, j+1 / 2}^{n+1}, \bar{w}_{i+1 / 2, j+1 / 2}^{n+1}, \overline{v w}_{i+1 / 2, j+1 / 2}^{n+1}\right\}_{i j},
$$

with respect to the dual mesh, one would need to accurately approximate the following quantities

$$
\bar{p}_{i+1 / 2, j+1 / 2}^{n}, \forall i, j, \text { with } p=u, v, w, v w, \quad u\left(x_{*}, y_{*}, t^{n}+\Delta t \theta_{m}\right), m=1,2,3, \text { and }\left(x_{*}, y_{*}\right) \in \mathcal{G} .
$$

Recall at $t^{n},\left\{\bar{u}_{i j}^{n}, \bar{v}_{i j}^{n}, \bar{w}_{i j}^{n}, \overline{v w}_{i j}^{n}\right\}_{i j}$ are available. To reconstruct the staggered moments $\left\{\bar{u}_{i+1 / 2, j+1 / 2}^{n}\right.$, $\left.\bar{v}_{i+1 / 2, j+1 / 2}^{n}, \bar{w}_{i+1 / 2, j+1 / 2}^{n}, \overline{v w}_{i+1 / 2, j+1 / 2}^{n}\right\}_{i j}$ in (3.19), one can apply the same fifth-order HWENO procedure described in Section 2.2.

To approximate the point values $u\left(x_{*}, y_{*}, t^{n}+\Delta t \theta_{m}\right)$ with $\left(x_{*}, y_{*}\right) \in \mathcal{G}, m=1,2,3$ in (3.19), we will apply the fourth-order NCE-RK method to an auxiliary ODE problem (3.1) starting from $t=t^{n}$, where $y(t)$ in (3.1) is the collection of $u\left(x_{*}, y_{*}, t\right), \forall\left(x_{*}, y_{*}\right) \in \mathcal{G}$, and $-H(t, y)$ in (3.1) is a vector valued functional which reconstructs $f_{x}\left(u\left(x_{*}, y_{*}, t\right)\right)+g_{y}\left(u\left(x_{*}, y_{*}, t\right)\right), \forall\left(x_{*}, y_{*}\right) \in \mathcal{G}$, based on $y(t)$, and is denoted as $\mathcal{R}(y(t))$. That is, $H(t, y(t))=-\mathcal{R}(y(t))$. To complete the description of this ODE problem, hence the strategy to approximate the point values $u\left(x_{*}, y_{*}, t^{n}+\Delta t \theta_{m}\right)$ in (3.19), two more ingredients are needed. One is the initial values 
$f\left(u\left(x_{*}, y_{*}, t^{n}\right)\right)$ and $g\left(u\left(x_{*}, y_{*}, t^{n}\right)\right)$, namely, $u\left(x_{*}, y_{*}, t^{n}\right), \forall\left(x_{*}, y_{*}\right) \in \mathcal{G}$, and it can be reconstructed with the fifth-order HWENO procedure based on the given moments $\left\{\bar{u}_{i j}^{n}, \bar{v}_{i j}^{n}, \bar{w}_{i j}^{n}, \overline{v w}_{i j}^{n}\right\}_{i j}$ on the primal mesh as presented in Section 2.2. The other ingredient is the functional $\mathcal{R}(y(t))$, which will be specified in the remaining of this subsection.

To reconstruct $f_{x}\left(u\left(x_{*}, y_{*}, t\right)\right), g_{y}\left(u\left(x_{*}, y_{*}, t\right)\right), \forall\left(x_{*}, y_{*}\right) \in \mathcal{G}$ based on $u\left(x_{*}, y_{*}, t\right), \forall\left(x_{*}, y_{*}\right) \in \mathcal{G}$, we propose a dimension-by-dimension procedure. From all the Gauss-Lobatto quadrature nodes $\mathcal{G}$ with respect to the dual mesh, we relabel those located in the cell $I_{i j}$ as $p_{i, j}^{1}, \cdots, p_{i, j}^{9}$, which are all interior nodes (also see Figure 2).

$$
\begin{aligned}
& p_{i, j}^{1}=\left(x_{i-\frac{1}{2}+\frac{\sqrt{5}}{10}}, \quad y_{j-\frac{1}{2}+\frac{\sqrt{5}}{10}}\right), \quad p_{i, j}^{2}=\left(x_{i}, y_{j-\frac{1}{2}+\frac{\sqrt{5}}{10}}\right), \quad p_{i, j}^{3}=\left(x_{i+\frac{1}{2}-\frac{\sqrt{5}}{10}}, y_{j-\frac{1}{2}+\frac{\sqrt{5}}{10}}\right), \\
& p_{i, j}^{4}=\left(x_{i-\frac{1}{2}+\frac{\sqrt{5}}{10}}, y_{j}\right), p_{i, j}^{5}=\left(x_{i}, y_{j}\right), p_{i, j}^{6}=\left(x_{i+\frac{1}{2}-\frac{\sqrt{5}}{10}}, y_{j}\right), \\
& p_{i, j}^{7}=\left(x_{i-\frac{1}{2}+\frac{\sqrt{5}}{10}}, y_{j+\frac{1}{2}-\frac{\sqrt{5}}{10}}\right), p_{i, j}^{8}=\left(x_{i}, y_{j+\frac{1}{2}-\frac{\sqrt{5}}{10}}\right), p_{i, j}^{9}=\left(x_{i+\frac{1}{2}-\frac{\sqrt{5}}{10}}, y_{j+\frac{1}{2}-\frac{\sqrt{5}}{10}}\right) .
\end{aligned}
$$

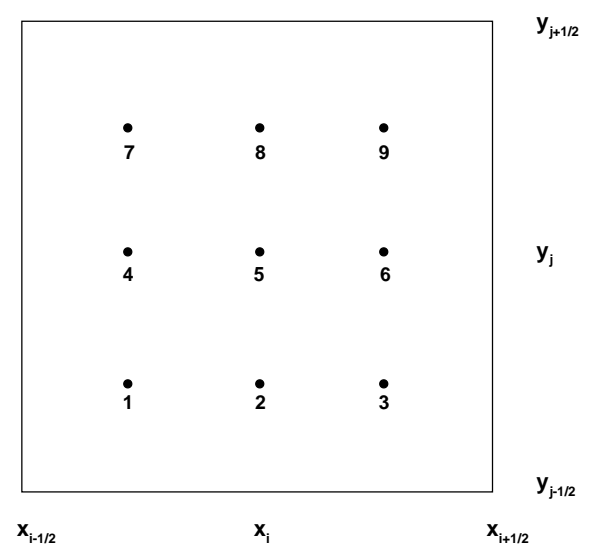

Figure 2: Two-dimensional Gauss-Lobatto quadrature nodes in $\mathcal{G}$ located within $I_{i j}$.

Now using the one-dimensional fifth-order accurate WENO reconstruction in Section 3.1, along $x$ direction,

- we reconstruct $\left\{f_{x}\left(u\left(p_{i, j}^{1}\right)\right), f_{x}\left(u\left(p_{i, j}^{2}\right)\right), f_{x}\left(u\left(p_{i, j}^{3}\right)\right)\right\}_{i j}$ based on

$$
\left\{f\left(u\left(p_{i-1, j}^{2}\right)\right), f\left(u\left(p_{i-1, j}^{3}\right)\right), f\left(u\left(p_{i, j}^{1}\right)\right), f\left(u\left(p_{i, j}^{2}\right)\right), f\left(u\left(p_{i, j}^{3}\right)\right), f\left(u\left(p_{i+1, j}^{1}\right)\right), f\left(u\left(p_{i+1, j}^{2}\right)\right)\right\}
$$

- we reconstruct $\left\{f_{x}\left(u\left(p_{i, j}^{4}\right)\right), f_{x}\left(u\left(p_{i, j}^{5}\right)\right), f_{x}\left(u\left(p_{i, j}^{6}\right)\right)\right\}_{i j}$ based on

$$
\left\{f\left(u\left(p_{i-1, j}^{5}\right)\right), f\left(u\left(p_{i-1, j}^{6}\right)\right), f\left(u\left(p_{i, j}^{4}\right)\right), f\left(u\left(p_{i, j}^{5}\right)\right), f\left(u\left(p_{i, j}^{6}\right)\right), f\left(u\left(p_{i+1, j}^{4}\right)\right), f\left(u\left(p_{i+1, j}^{5}\right)\right)\right\} ;
$$

- and we reconstruct $\left\{f_{x}\left(u\left(p_{i, j}^{7}\right)\right), f_{x}\left(u\left(p_{i, j}^{8}\right)\right), f_{x}\left(u\left(p_{i, j}^{9}\right)\right)\right\}_{i j}$ based on

$$
\left\{f\left(u\left(p_{i-1, j}^{8}\right)\right), f\left(u\left(p_{i-1, j}^{9}\right)\right), f\left(u\left(p_{i, j}^{7}\right)\right), f\left(u\left(p_{i, j}^{8}\right)\right), f\left(u\left(p_{i, j}^{9}\right)\right), f\left(u\left(p_{i+1, j}^{7}\right)\right), f\left(u\left(p_{i+1, j}^{8}\right)\right)\right\} .
$$

Here and below the dependance on $t$ is omitted in $u$. Along $y$-direction, 
- we reconstruct $\left\{g_{y}\left(u\left(p_{i, j}^{1}\right)\right), g_{y}\left(u\left(p_{i, j}^{4}\right)\right), g_{y}\left(u\left(p_{i, j}^{7}\right)\right)\right\}_{i j}$ based on

$$
\left\{g\left(u\left(p_{i, j-1}^{4}\right)\right), g\left(u\left(p_{i, j-1}^{7}\right)\right), g\left(u\left(p_{i, j}^{1}\right)\right), g\left(u\left(p_{i, j}^{4}\right)\right), g\left(u\left(p_{i, j}^{7}\right)\right), g\left(u\left(p_{i, j+1}^{1}\right)\right), g\left(u\left(p_{i, j+1}^{4}\right)\right)\right\} ;
$$

- we reconstruct $\left\{g_{y}\left(u\left(p_{i, j}^{2}\right)\right), g_{y}\left(u\left(p_{i, j}^{5}\right)\right), g_{y}\left(u\left(p_{i, j}^{8}\right)\right)\right\}_{i j}$ based on

$$
\left\{g\left(u\left(p_{i, j-1}^{5}\right)\right), g\left(u\left(p_{i, j-1}^{8}\right)\right), g\left(u\left(p_{i, j}^{2}\right)\right), g\left(u\left(p_{i, j}^{5}\right)\right), g\left(u\left(p_{i, j}^{8}\right)\right), g\left(u\left(p_{i, j+1}^{2}\right)\right), g\left(u\left(p_{i, j+1}^{5}\right)\right)\right\} ;
$$

- and we reconstruct $\left\{g_{y}\left(u\left(p_{i, j}^{3}\right)\right), g_{y}\left(u\left(p_{i, j}^{6}\right)\right), g_{y}\left(u\left(p_{i, j}^{9}\right)\right)\right\}_{i j}$ based on

$$
\left\{g\left(u\left(p_{i, j-1}^{6}\right)\right), g\left(u\left(p_{i, j-1}^{9}\right)\right), g\left(u\left(p_{i, j}^{3}\right)\right), g\left(u\left(p_{i, j}^{6}\right)\right), g\left(u\left(p_{i, j}^{9}\right)\right), g\left(u\left(p_{i, j+1}^{3}\right)\right), g\left(u\left(p_{i, j+1}^{6}\right)\right)\right\} .
$$

The algorithms presented in this and previous subsections can be extended to the system of conservation laws, see also Section 2.

\section{Numerical examples}

In this section, we will present a set of numerical experiments to illustrate the high-order accuracy and the robustness of the proposed methods to simulate one- and two-dimensional scalar or a system of hyperbolic conservation laws. The solutions can be either smooth or non-smooth. The numerical results are obtained by the proposed fifth-order central HWENO scheme with the third-order Lax-Wendroff method (C-HWENO5-LW3) or with the fourth-order NCE-RK method (C-HWENO5-NCERK4) in time for both one and two dimensions. Uniform meshes with $N$ elements and $N_{x} \times N_{y}$ elements are used in one and two dimensions, respectively.

The time step $\Delta t$ is dynamically chosen. In particular, in one-dimensional scalar case, we take

$$
\Delta t=C_{c f l} \frac{\Delta x}{\max \left|f^{\prime}(u)\right|},
$$

and in two-dimensional scalar case, we have

$$
\Delta t=\frac{C_{c f l}}{\left(\max \left|f^{\prime}(u)\right| / \Delta x+\max \left|g^{\prime}(u)\right| / \Delta y\right)} .
$$

The CFL number $C_{c f l}$ is taken as 0.25 for one-dimensional tests, and 0.2 for two-dimensional ones except for some accuracy tests when the time step needs to be adjusted properly to ensure the spatial errors dominate. In the system case, $\left|f^{\prime}(u)\right|$ and $\left|g^{\prime}(u)\right|$ above are replaced by the eigenvalue of the Jacobian of $f(u)$ and $g(u)$, respectively, with the largest absolute value.

\subsection{Accuracy tests with smooth solutions}

We first validate the accuracy of the proposed schemes when the solutions are smooth. The conservation laws can be scalar, or system in one and two dimensions.

Example 4.1. We consider the one-dimensional Burgers' equation, which is scalar and nonlinear,

$$
u_{t}+\left(\frac{u^{2}}{2}\right)_{x}=0
$$

with the initial condition $u(x, 0)=0.5+\sin (\pi x)$, and a 2-periodic boundary condition. We take the CFL number $C_{c f l}=0.1$. When $t=0.5 / \pi$, the solution is still smooth, and the $L_{1}$ and $L_{\infty}$ errors and numerical 
orders of accuracy are presented in Table 1 for C-HWENO5-LW3 and C-HWENO5-NCERK4 schemes. We can see that both schemes achieve their designed fifth-order accuracy with comparable errors. Compared with the numerical results by the C-HWENO scheme previously proposed in [34], the numerical solution by the newly proposed scheme in this paper has smaller errors on the same computational mesh.

Table 1: Burgers' equation $u_{t}+\left(u^{2} / 2\right)_{x}=0$, with $u(x, 0)=0.5+\sin (\pi x)$, and a periodic boundary condition. C-HWENO5-LW3 and C-HWENO5-NCERK4. $t=0.5 / \pi . L_{1}$ and $L_{\infty}$ errors and orders of accuracy.

\begin{tabular}{|c|c|c|c|c|c|c|c|c|}
\hline \multirow[t]{2}{*}{$\bar{N}$} & \multicolumn{4}{|c|}{ C-HWENO5-LW3 } & \multicolumn{4}{|c|}{ C-HWENO5-NCERK4 } \\
\hline & $L_{1}$ error & Order & $L_{\infty}$ error & Order & $L_{1}$ error & Order & $L_{\infty}$ error & Order \\
\hline 10 & $1.11 \mathrm{e}-02$ & & $3.05 \mathrm{e}-02$ & & $1.09 \mathrm{E}-02$ & & $3.20 \mathrm{E}-02$ & \\
\hline 20 & $1.57 \mathrm{e}-03$ & 2.82 & $1.12 \mathrm{e}-02$ & 1.45 & $1.56 \mathrm{E}-03$ & 2.81 & $1.11 \mathrm{E}-02$ & 1.53 \\
\hline 40 & $9.91 \mathrm{e}-05$ & 3.99 & $9.12 \mathrm{e}-04$ & 3.62 & $9.58 \mathrm{E}-05$ & 4.02 & $9.05 \mathrm{E}-04$ & 3.62 \\
\hline 80 & $4.41 \mathrm{e}-06$ & 4.49 & $3.82 \mathrm{e}-05$ & 4.58 & $4.29 \mathrm{E}-06$ & 4.48 & $3.67 \mathrm{E}-05$ & 4.63 \\
\hline 160 & $1.72 \mathrm{e}-07$ & 4.68 & $1.82 \mathrm{e}-06$ & 4.39 & $1.68 \mathrm{E}-07$ & 4.67 & $1.70 \mathrm{E}-06$ & 4.43 \\
\hline 320 & $4.91 \mathrm{e}-09$ & 5.13 & $6.02 \mathrm{e}-08$ & 4.92 & $4.92 \mathrm{E}-09$ & 5.10 & $5.80 \mathrm{E}-08$ & 4.87 \\
\hline 640 & $1.48 \mathrm{e}-10$ & 5.05 & $1.12 \mathrm{e}-09$ & 5.75 & $1.20 \mathrm{E}-10$ & 5.36 & $1.22 \mathrm{E}-09$ & 5.57 \\
\hline
\end{tabular}

Example 4.2. We consider the one-dimensional nonlinear system of Euler equations

$$
u_{t}+f(u)_{x}=0
$$

with

$$
u=(\rho, \rho v, E)^{T}, \quad f(u)=\left(\rho v, \rho v^{2}+p, v(E+p)\right)^{T} .
$$

Here $\rho$ is the density, $v$ is the velocity, $E$ is the total energy, $p$ is the pressure which is related to the conservative quantities through the relation $E=\frac{p}{\gamma-1}+\frac{1}{2} \rho v^{2}$, and $\gamma=1.4$. The initial condition is set to be $\rho(x, 0)=1+0.2 \sin (\pi x), v(x, 0)=1, p(x, 0)=1$, with a 2-periodic boundary condition. The exact solution is $\rho(x, t)=1+0.2 \sin (\pi(x-t)), v(x, t)=1$, and $p(x, t)=1$. We compute the solution up to $t=2$ with the CFL number $C_{c f l}=0.1$. The $L_{1}$ and $L_{\infty}$ errors and numerical orders of accuracy for the density $\rho$ are shown in Table 2 for C-HWENO5-LW3 and C-HWENO5-NCERK4 schemes. Both schemes achieve their designed fifth-order accuracy with comparable errors. Again compared with the numerical results by the C-HWENO scheme previously proposed in [34], the numerical solution by the newly proposed scheme in this paper has smaller errors on the same computational mesh.

Example 4.3. We consider the nonlinear Burgers' equation in two dimensions

$$
u_{t}+\left(\frac{u^{2}}{2}\right)_{x}+\left(\frac{u^{2}}{2}\right)_{y}=0
$$

with the initial condition $u(x, y, 0)=0.5+\sin (\pi(x+y) / 2)$, and a 4-periodic boundary condition in each direction. When $t=0.5 / \pi$ the solution is still smooth. The CFL number is taken as $C_{c f l}=0.1$ and the $L_{1}$ and $L_{\infty}$ errors and numerical orders of accuracy are presented in Table 3 for C-HWENO5-LW3 and C-HWENO5-NCERK4 schemes. Both schemes achieve their designed fifth-order accuracy with comparable errors. Again compared with the numerical results by the C-HWENO scheme previously proposed in [34], the numerical solution by the newly proposed scheme in this paper has smaller errors on the same computational mesh. 
Table 2: Euler equations, with $\rho(x, 0)=1+0.2 \sin (\pi x), v(x, 0)=1, p(x, 0)=1$, and periodic boundary conditions. C-HWENO5-LW3 and C-HWENO5-NCERK4. $t=2$. $L_{1}$ and $L_{\infty}$ errors and orders of accuracy of density $\rho$.

\begin{tabular}{|c|c|c|c|c|c|c|c|c|}
\hline \multirow[t]{2}{*}{$N$} & \multicolumn{4}{|c|}{ C-HWENO5-LW3 } & \multicolumn{4}{|c|}{ C-HWENO5-NCERK4 } \\
\hline & $L_{1}$ error & Order & $L_{\infty}$ error & Order & $L_{1}$ error & Order & $L_{\infty}$ error & Order \\
\hline 10 & $1.55 \mathrm{e}-02$ & & $2.37 \mathrm{e}-02$ & & $1.53 \mathrm{E}-02$ & & $2.34 \mathrm{E}-02$ & \\
\hline 20 & $7.27 \mathrm{e}-04$ & 4.41 & $1.15 \mathrm{e}-03$ & 4.37 & $7.20 \mathrm{E}-04$ & 4.41 & $1.14 \mathrm{E}-03$ & 4.35 \\
\hline 40 & $2.25 \mathrm{e}-05$ & 5.01 & $4.13 \mathrm{e}-05$ & 4.80 & $2.22 \mathrm{E}-05$ & 5.02 & $4.08 \mathrm{E}-05$ & 4.80 \\
\hline 80 & $7.01 \mathrm{e}-07$ & 5.00 & $1.36 \mathrm{e}-06$ & 4.92 & $6.90 \mathrm{E}-07$ & 5.01 & $1.34 \mathrm{E}-06$ & 4.93 \\
\hline 160 & $2.20 \mathrm{e}-08$ & 4.99 & $4.16 \mathrm{e}-08$ & 5.03 & $2.16 \mathrm{E}-08$ & 5.00 & $4.08 \mathrm{E}-08$ & 5.04 \\
\hline 320 & $6.82 \mathrm{e}-10$ & 5.01 & $1.18 \mathrm{e}-09$ & 5.14 & $6.53 \mathrm{E}-10$ & 5.05 & 1.13E-09 & 5.17 \\
\hline 640 & $2.02 \mathrm{e}-11$ & 5.08 & $3.33 \mathrm{e}-11$ & 5.15 & $1.75 \mathrm{E}-11$ & 5.22 & $2.96 \mathrm{E}-11$ & 5.26 \\
\hline
\end{tabular}

Table 3: Burgers' equation $u_{t}+\left(u^{2} / 2\right)_{x}+\left(u^{2} / 2\right)_{y}=0$, with $u(x, y, 0)=0.5+\sin (\pi(x+y) / 2)$, and periodic boundary conditions. C-HWENO5-LW3 and C-HWENO5-NCERK4. $t=0.5 / \pi . L_{1}$ and $L_{\infty}$ errors and orders of accuracy.

\begin{tabular}{|c|c|c|c|c|c|c|c|c|}
\hline \multirow[t]{2}{*}{$\overline{N_{x} \times N_{y}}$} & \multicolumn{4}{|c|}{ C-HWENO5-LW3 } & \multicolumn{4}{|c|}{ C-HWENO5-NCERK4 } \\
\hline & $L_{1}$ error & Order & $L_{\infty}$ error & Order & $L_{1}$ error & Order & $L_{\infty}$ error & Order \\
\hline $10 \times 10$ & $2.11 \mathrm{e}-02$ & & $5.83 \mathrm{e}-02$ & & $2.11 \mathrm{E}-02$ & & $5.84 \mathrm{E}-02$ & \\
\hline $20 \times 20$ & $2.09 \mathrm{e}-03$ & 3.34 & $1.39 \mathrm{e}-02$ & 2.07 & $2.08 \mathrm{E}-03$ & 3.34 & 1.39E-02 & 2.07 \\
\hline $40 \times 40$ & $1.87 \mathrm{e}-04$ & 3.48 & $1.45 \mathrm{e}-03$ & 3.26 & $1.86 \mathrm{E}-04$ & 3.48 & $1.44 \mathrm{E}-03$ & 3.27 \\
\hline $80 \times 80$ & $8.39 \mathrm{e}-06$ & 4.48 & $7.48 \mathrm{e}-05$ & 4.28 & $8.29 \mathrm{E}-06$ & 4.49 & $7.48 \mathrm{E}-05$ & 4.27 \\
\hline $160 \times 160$ & $3.16 \mathrm{e}-07$ & 4.73 & $2.64 \mathrm{e}-06$ & 4.82 & $3.15 \mathrm{E}-07$ & 4.72 & $2.60 \mathrm{E}-06$ & 4.85 \\
\hline $320 \times 320$ & $8.64 \mathrm{e}-09$ & 5.19 & $7.87 \mathrm{e}-08$ & 5.07 & $8.72 \mathrm{E}-09$ & 5.17 & $7.78 \mathrm{E}-08$ & 5.06 \\
\hline $640 \times 640$ & $2.39 \mathrm{e}-10$ & 5.18 & $2.31 \mathrm{e}-09$ & 5.09 & $2.21 \mathrm{E}-10$ & 5.30 & $2.44 \mathrm{E}-09$ & 4.99 \\
\hline
\end{tabular}

Example 4.4. We here consider the nonlinear system of Euler equations in two dimensions

$$
U_{t}+f(U)_{x}+g(U)_{y}=0
$$

with

$$
\begin{gathered}
U=(\rho, \rho u, \rho v, E)^{T}, \quad f(U)=\left(\rho u, \rho u^{2}+p, \rho u v, u(E+p)\right)^{T}, \\
g(U)=\left(\rho v, \rho u v, \rho v^{2}+p, v(E+p)\right)^{T} .
\end{gathered}
$$

Here $\rho$ is the density, $(u, v)^{T}$ is the velocity, $E$ is the total energy, $p$ is the pressure which is related to the conservative quantities through the relation $E=\frac{p}{\gamma-1}+\frac{1}{2} \rho\left(u^{2}+v^{2}\right)$, and $\gamma=1.4$. The initial condition is set to be $\rho(x, y, 0)=1+0.2 \sin (\pi(x+y)), u(x, y, 0)=0.7, v(x, y, 0)=0.3, p(x, y, 0)=1$, with a 2-periodic boundary condition in each direction. The exact solution is $\rho(x, y, t)=1+0.2 \sin (\pi(x+y-(u+v) t))$, $u(x, y, t)=0.7, v(x, y, t)=0.3$, and $p(x, y, t)=1$. We compute the solution up to $t=2$ with the CFL number $C_{c f l}=0.1$. The $L_{1}$ and $L_{\infty}$ errors and numerical orders of accuracy of density $\rho$ are reported in Table 4 for C-HWENO5-LW3 and C-HWENO5-NCERK4 schemes. Both schemes achieve their designed fifth-order accuracy with comparable errors. Again compared with the numerical results by the C-HWENO scheme previously proposed in [34], the numerical solution by the newly proposed scheme in this paper has smaller errors on the same computational mesh. 
Table 4: Euler equations, with $\rho(x, y, 0)=1+0.2 \sin (\pi(x+y)), u(x, y, 0)=0.7, v(x, y, 0)=0.3, p(x, y, 0)=1$, and periodic boundary conditions. C-HWENO5-LW3 and C-HWENO5-NCERK4. $t=2 . L_{1}$ and $L_{\infty}$ errors and orders of accuracy of density $\rho$.

\begin{tabular}{|c|c|c|c|c|c|c|c|c|}
\hline \multirow[t]{2}{*}{$\overline{N_{x} \times N_{y}}$} & \multicolumn{4}{|c|}{ C-HWENO5-LW3 } & \multicolumn{4}{|c|}{ C-HWENO5-NCERK4 } \\
\hline & $L_{1}$ error & Order & $L_{\infty}$ error & Order & $L_{1}$ error & Order & $L_{\infty}$ error & Order \\
\hline $10 \times 10$ & $4.13 \mathrm{e}-02$ & & $6.26 \mathrm{e}-02$ & & $4.13 \mathrm{E}-02$ & & $6.26 \mathrm{E}-02$ & \\
\hline $20 \times 20$ & $2.20 \mathrm{e}-03$ & 4.23 & $3.02 \mathrm{e}-03$ & 4.37 & $2.20 \mathrm{E}-03$ & 4.23 & $3.02 \mathrm{E}-03$ & 4.37 \\
\hline $40 \times 40$ & $7.33 \mathrm{e}-05$ & 4.91 & $1.17 \mathrm{e}-04$ & 4.69 & $7.32 \mathrm{E}-05$ & 4.91 & $1.17 \mathrm{E}-04$ & 4.69 \\
\hline $80 \times 80$ & $2.25 \mathrm{e}-06$ & 5.03 & $3.98 \mathrm{e}-06$ & 4.88 & $2.25 \mathrm{E}-06$ & 5.02 & $3.98 \mathrm{E}-06$ & 4.88 \\
\hline $160 \times 160$ & $6.85 \mathrm{e}-08$ & 5.04 & $1.20 \mathrm{e}-07$ & 5.05 & $6.83 \mathrm{E}-08$ & 5.04 & $1.20 \mathrm{E}-07$ & 5.05 \\
\hline $320 \times 320$ & $1.95 \mathrm{e}-09$ & 5.13 & $3.20 \mathrm{e}-09$ & 5.23 & $1.94 \mathrm{E}-09$ & 5.14 & 3.19E-09 & 5.23 \\
\hline $640 \times 640$ & $4.68 \mathrm{e}-11$ & 5.38 & $7.99 \mathrm{e}-11$ & 5.32 & $4.61 \mathrm{E}-11$ & 5.39 & $7.85 \mathrm{E}-11$ & 5.34 \\
\hline
\end{tabular}

\subsection{Test cases with non-smooth solutions}

We now test the performance of the proposed methods in terms of their resolution and non-oscillatory property when solving problems with non-smooth features, such as shocks, rarefaction, or contact discontinuity, in their solutions.

Example 4.5. We consider the same one-dimensional nonlinear Burgers' equation (4.1) as in Example 4.1 with the same initial and boundary conditions, except that we now present the numerical solutions at $t=1.5 / \pi$ after a shock forms. In Figure 3, the solutions of C-HWENO5-LW3 and C-HWENO5-NCERK4 schemes are plotted, together with the exact solution. The mesh is uniform with $N=80$ elements. We can see that both schemes lead to non-oscillatory shock transitions in the solutions.

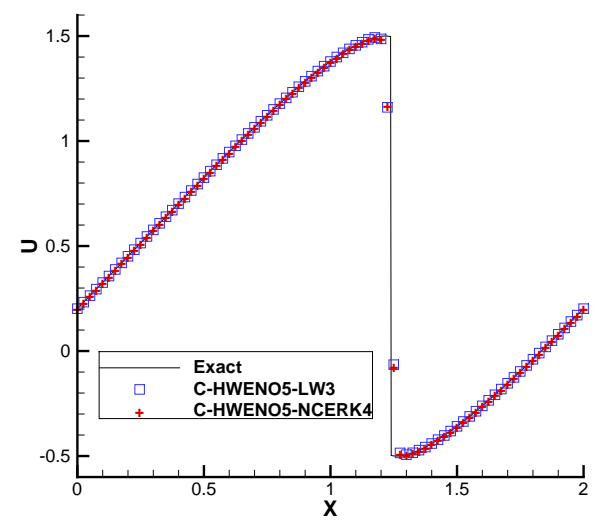

Figure 3: Burgers' equation in one dimension. $u(x, 0)=0.5+\sin (\pi x) . t=1.5 / \pi$ and $N=80$. Solid line: exact solution; square: C-HWENO5-LW3; plus: C-HWENO5-NCERK4.

Example 4.6. We here consider a one-dimensional nonlinear non-convex scalar Buckley-Leverett problem

$$
u_{t}+\left(\frac{4 u^{2}}{4 u^{2}+(1-u)^{2}}\right)_{x}=0
$$


with the initial condition: $u=1$ when $-\frac{1}{2} \leq x \leq 0$ and $u=0$ elsewhere. The computational domain is $[-1,1]$, with constant boundary conditions applied to both ends. The solution is computed up to $t=0.4$. The exact solution contains shock, rarefaction, and a contact discontinuity. We remark that some high-order schemes may fail to converge to the correct entropy solution for this problem. In Figure 4, the solutions of C-HWENO5-LW3 and C-HWENO5-NCERK4 schemes are shown with $N=80$ mesh elements, together with the exact solution. One can see both schemes capture the correct entropy solution well, with good resolutions for all the major features in the solution.

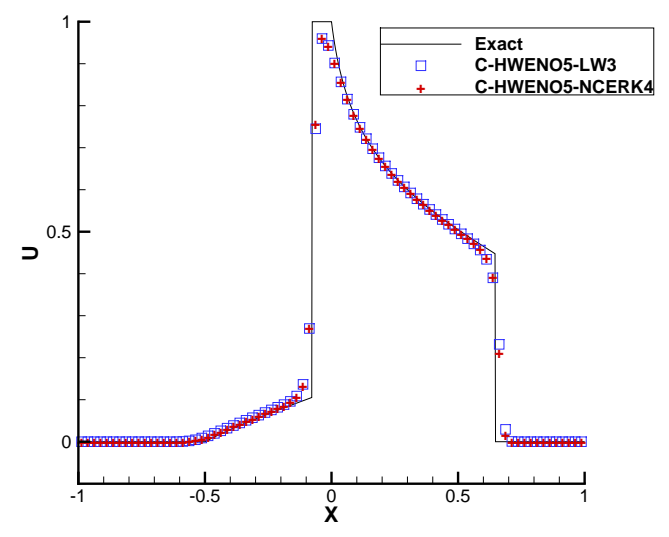

Figure 4: The Buckley-Leverett problem. $t=0.4$ and $N=80$. Solid line: exact solution; square: CHWENO5-LW3; plus: C-HWENO5-NCERK4.

Example 4.7. We solve the one-dimensional Euler equations (4.2) with a Riemann initial condition for the Lax problem

$$
(\rho, v, p)=(0.445,0.698,3.528) \quad \text { for } x \leq 0, \quad(\rho, v, p)=(0.5,0,0.571) \quad \text { for } x>0 .
$$

The computational domain is $[-5,5]$, with inflow/outflow boundary conditions applied to left/right ends. The schemes are run up to $t=1.3$. In Figure 5 , we plot the computed density $\rho$ with $N=200$ mesh elements by C-HWENO5-LW3 and C-HWENO5-NCERK4 schemes, together with the exact solution. One can see that both schemes render equally good non-oscillatory shock transitions for this problem.

Example 4.8. The non-smooth examples we have presented so far contain simple smooth regions in the solutions, for which capturing the shock sharply with non-oscillatory transitions is the main focus and usually a good second-order non-oscillatory scheme would give satisfactory results. The examples presented here are mainly to demonstrate the non-oscillatory properties of the proposed high-order schemes. A highorder scheme would be more advantageous when the solution contains both shocks and complex smooth structures. One such example is the Shu-Osher problem, which describes shock interacting with entropy waves [32]. This example is modeled by the one-dimensional Euler equations (4.2) with a moving Mach-3 shock interacting with sine waves in density, i.e. initially

$$
\begin{aligned}
& (\rho, v, p)=(3.857143,2.629369,10.333333) \quad \text { for } x<-4, \\
& (\rho, v, p)=(1+\sigma \sin 5 x, 0,1) \quad \text { for } x \geq-4 .
\end{aligned}
$$




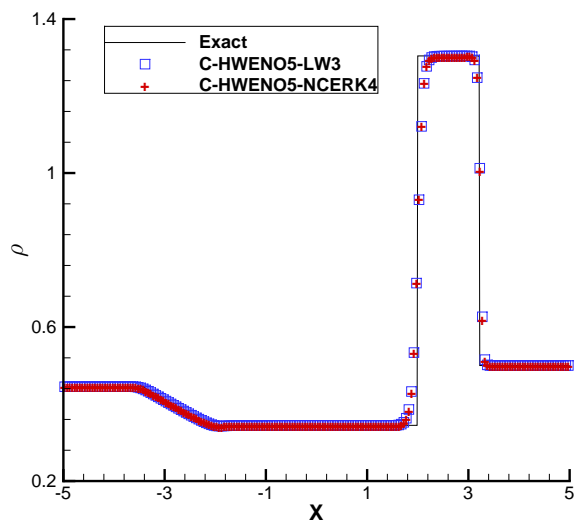

Figure 5: The Lax problem. $t=1.3$ and $N=200$. Density $\rho$. Solid line: exact solution; square: CHWENO5-LW3; plus: C-HWENO5-NCERK4.

The computational domain is $[-5,5]$, and the boundary conditions are taken to be the same as the initial data. Here we take $\sigma=0.2$. In Figure 6 , we plot the computed density $\rho$ at $t=1.8$ by C-HWENO5LW3 and C-HWENO5-NCERK4 schemes with $N=400$ mesh elements, together with a reference solution obtained by the fifth-order finite difference WENO scheme [15] with 16000 grid points, we further zoom in the oscillatory region of the solution between $x=0.5$ and $x=2.5$. One can see that this example very well demonstrates the good resolution of the proposed methods to resolve smooth features in the solution as well as the non-oscillatory nature of the methods to capture discontinuities.
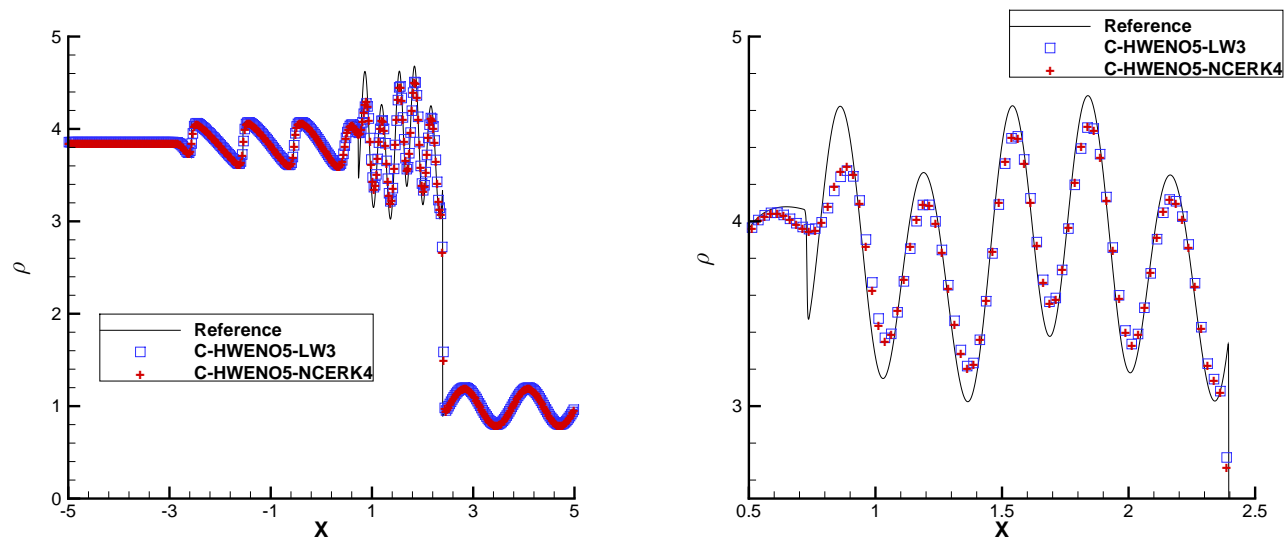

Figure 6: The shock density wave interaction problem. $t=1.8$ and $N=400$. Density $\rho$. Solid line: reference solution; square: C-HWENO5-LW3; plus: C-HWENO5-NCERK4.

Example 4.9. We here consider the interaction of blast waves, modeled by the one-dimensional Euler 
equations (4.2) with the initial condition

$$
\begin{aligned}
& (\rho, v, p)=(1,0,1000) \quad \text { for } 0 \leq x<0.1 \\
& (\rho, v, p)=(1,0,0.01) \quad \text { for } 0.1 \leq x<0.9 \\
& (\rho, v, p)=(1,0,100) \quad \text { for } 0.9 \leq x
\end{aligned}
$$

The computational domain is $[0,1]$, with reflecting boundary conditions applied to both ends, see $[11,36]$. In Figure 7, we plot the computed density $\rho$ at $t=0.038$ by C-HWENO5-LW3 and C-HWENO5-NCERK4 schemes with $N=800$ mesh elements, together with a reference solution obtained by the fifth-order finite difference WENO scheme [15] with 16000 grid points. We can see that both schemes give equally good resolution for this problem.

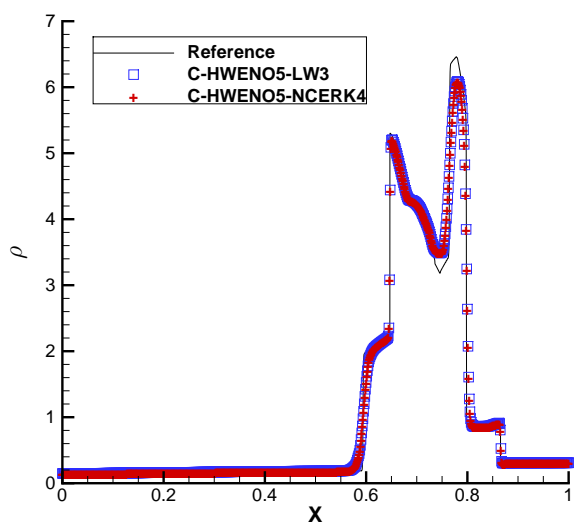

Figure 7: The interaction of blast waves problem. $t=0.038$ and $N=800$. Density $\rho$. Solid line: reference solution; square: C-HWENO5-LW3; plus: C-HWENO5-NCERK4.

Example 4.10. We solve the same two-dimensional Burgers' equation (4.3) as in Example 4.3 with the same initial and boundary conditions, except that we now present the numerical solutions at $t=1.5 / \pi$ after a shock forms. In Figure 8, we plot a slice of the computed solution at $x=y$ on a $80 \times 80$ mesh by C-HWENO5-LW3 and C-HWENO5-NCERK4 schemes, together with the exact solution. The surfaces of the solutions computed by C-HWENO5-LW3 and C-HWENO5-NCERK4 schemes are also shown. Both schemes render good non-oscillatory shock transitions for this problem.

Example 4.11. We here consider the example of double Mach reflection, which is originally from [36] and modeled by the two-dimensional Euler equations (4.4). The computational domain is $[0,4] \times[0,1]$. The reflecting wall lies at the bottom, starting from $x=\frac{1}{6}$. Initially a right-moving Mach-10 shock is positioned at $x=\frac{1}{6}, y=0$ and makes a $60^{\circ}$ angle with $x$-axis. For the bottom boundary, the exact post-shock condition is imposed for the part from $x=0$ to $x=\frac{1}{6}$, and a reflective boundary condition is used for the rest. At the top boundary, the flow values are set to describe the exact motion of a Mach-10 shock. Post-shock and pre-shock conditions are imposed for the left and right boundary conditions, respectively. We compute up to $t=0.2$. We use four different uniform meshes, with $240 \times 60,480 \times 120,960 \times 240,1920 \times 480$ mesh elements. We only show the results with $1920 \times 480$ mesh elements to save space. In Figure 9 , the density contour plots are presented in the region of $[0,3] \times[0,1]$ by C-HWENO5-LW3 and C-HWENO5-NCERK4 schemes, 

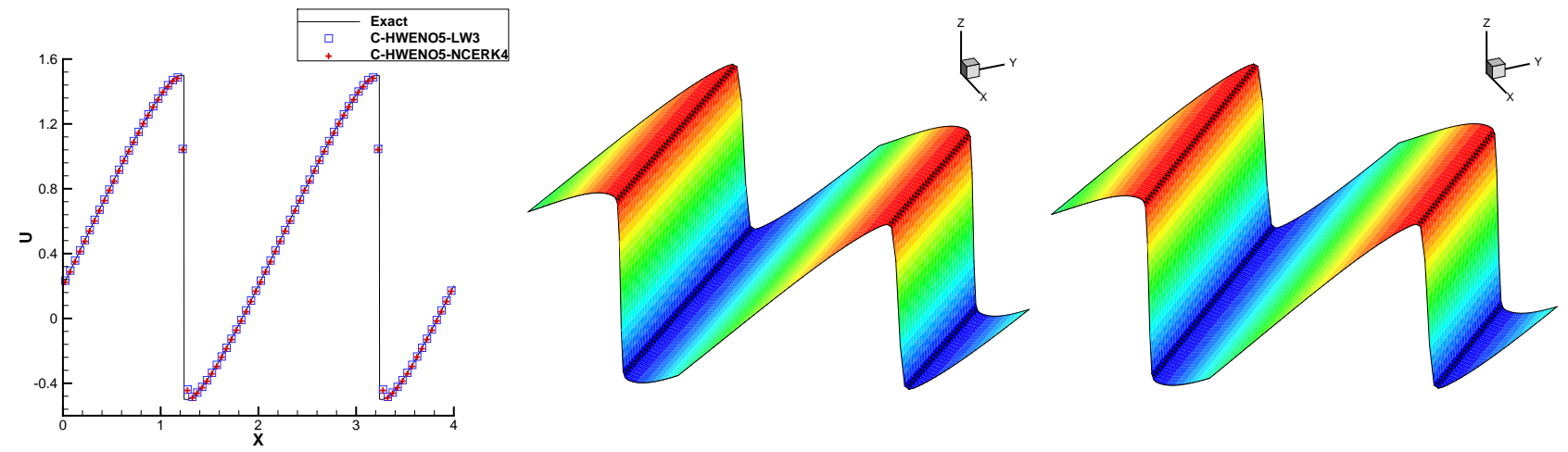

Figure 8: Burgers' equation in two dimensions. $u(x, y, 0)=0.5+\sin (\pi(x+y) / 2) \cdot t=1.5 / \pi$ and $N_{x} \times N_{y}=$ $80 \times 80$. A slide of the solution (left) at $x=y$, solid line: exact solution; square: C-HWENO5-LW3; plus: C-HWENO5-NCERK4. The surface of solution computed by C-HWENO5-LW3 (middle) and C-HWENO5NCERK4 (right).

respectively. Both schemes show good resolution to approximate the solution with comparable results. All contour plots are with 30 equally-spaced contour lines for the density from 1.5 to 22.7.
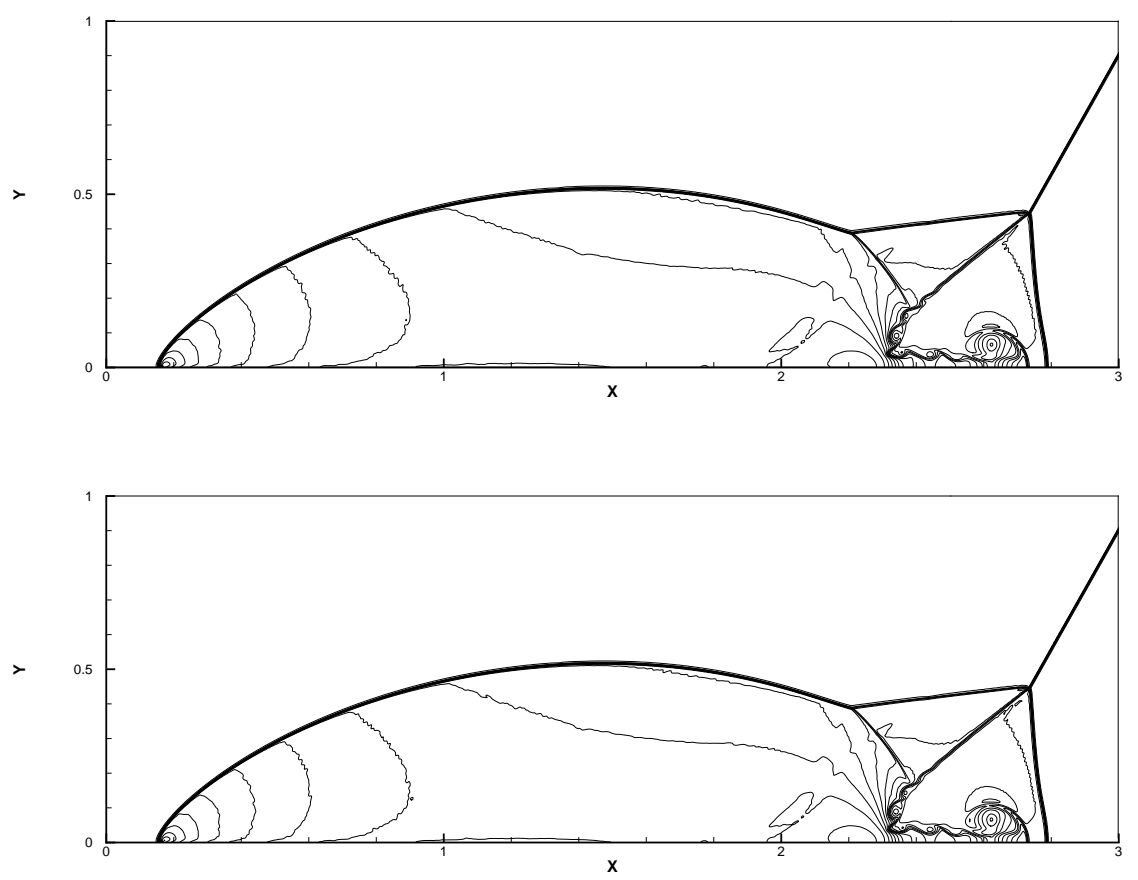

Figure 9: Double Mach reflection problem. $t=0.2$ and $N_{x} \times N_{y}=1920 \times 480$. C-HWENO5-LW3 (top) and C-HWENO5-NCERK4 (bottom). 30 equally spaced density contours from 1.5 to 22.7.

Example 4.12. Our final example is about a Mach-3 wind tunnel with a step. It is from [36] and modeled by the two-dimensional Euler equations (4.4). The setup of the problem is as follows. The wind 
tunnel is 1 length unit wide and 3 length units long. The step is 0.2 length units high and is located 0.6 length units from the left end of the tunnel. The problem is initialized by a right-going Mach-3 flow. Reflective boundary conditions are applied along the walls of the tunnel, and inflow/outflow boundary conditions are applied at the entrance/exit. The corner of the step is a singular point and we treat it as the same way as in [36], which is based on the assumption of a nearly steady flow in the region near the corner. We compute up to $t=4$. We use four different uniform meshes, with $120 \times 40,240 \times 80,480 \times 160,960 \times 320$ mesh elements. Again, we only show the results with $960 \times 320$ mesh elements to save space. In Figure 10, the density contour plots are presented by C-HWENO5-LW3 and C-HWENO5-NCERK4 schemes, respectively. Both schemes show good resolution to approximate the solution with comparable results. All contour plots are with 30 equally-spaced contour lines for the density from 0.32 to 6.15 .
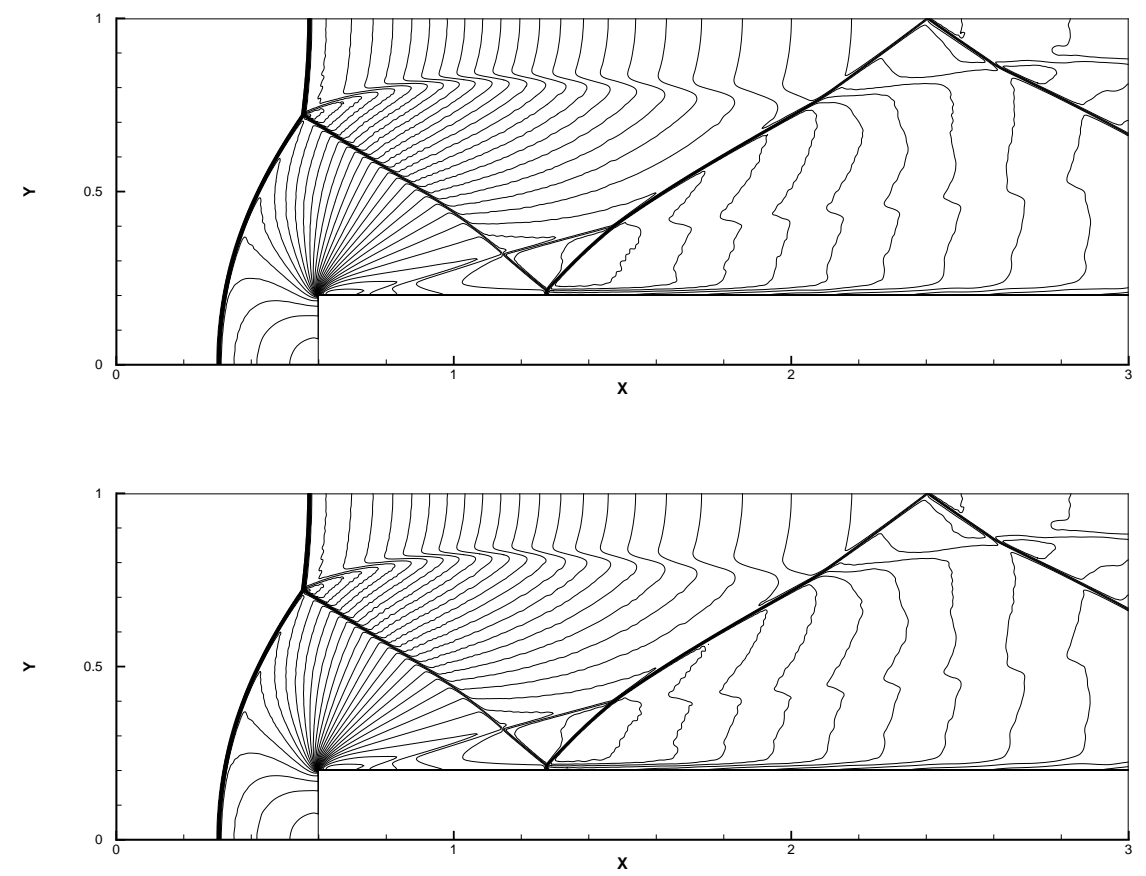

Figure 10: Forward step problem. $t=4$ and $N_{x} \times N_{y}=960 \times 320$. C-HWENO5-LW3 (top) and C-HWENO5NCERK4 (bottom). 30 equally spaced density contours from 0.32 to 6.15 .

We want to point out that for both example 4.11 and example 4.12, the proposed methods in this paper produce less oscillatory numerical solutions than our previously developed methods in [34] when the meshes are very refined, implying the new methods here have better numerical robustness.

We also compare the computational times of the previous C-HWENO methods in [34] and the newly proposed C-HWENO methods when they are applied to three two-dimensional examples. In Table 5, the computational times are presented for example 4.4 (two-dimensional Euler equations with smooth solution) with $160 \times 160$ mesh elements, example 4.11 (double Mach reflection problem) with $960 \times 240$ mesh elements, and example 4.12 (forward step problem) with $480 \times 160$ mesh elements, respectively. One can observe from this experiment that the new methods proposed in this paper are computationally more efficient. 
Table 5: The computational times (in seconds) of the C-HWENO methods in [34] and the new C-HWENO methods in this paper for three two-dimensional examples.

\begin{tabular}{llllll}
\hline Problem & \multicolumn{2}{l}{ C-HWENO methods in [34] } & & \multicolumn{2}{c}{ new C-HWENO methods } \\
\cline { 2 - 3 } \cline { 6 - 6 } & Lax-Wendroff & NCE of RK & & Lax-Wendroff & NCE of RK \\
\hline Example 4.4 & 5431 & 6856 & & 3323 & 2941 \\
Example 4.11 & 153384 & 159950 & & 79681 & 61901 \\
Example 4.12 & 163829 & 171102 & & 86065 & 70985 \\
\hline
\end{tabular}

\section{Concluding remarks}

In this paper, we design a new class of high-order central Hermite WENO schemes for solving one- and two-dimensional hyperbolic conservation laws. The methods use Hermite WENO reconstruction based on moments of the solution to discretize in space, and Lax-Wendroff type methods or the natural continuous extension of Runge-Kutta methods as time discretizations, in a central finite volume framework on staggered meshes. Our schemes evolve in time the moments of the solution rather than the solution and its derivative(s) as in [34] for the spatial reconstruction. As central HWENO schemes, our proposed methods have the advantages of being compact in reconstruction and requiring no flux splitting or numerical fluxes. In the system case, local characteristic decomposition is applied in the reconstruction of half-cell (or quartercell) moments to achieve better non-oscillatory properties of the schemes, meanwhile maintaining good computational efficiency. Instead of working with staggered meshes, the proposed spatial reconstructions can also be applied to the central finite volume schemes defined on two overlapping meshes [23].

The Hermite WENO reconstruction examined in this paper, that involves zeroth-order and first-order moments of the solution, requires more storage than the regular WENO reconstruction. One major advantage of our schemes is that the two-dimensional HWENO spatial reconstruction can be implemented through a dimension-by-dimension strategy, while the reconstruction in [34] is truly two-dimensional. In other words, our proposed HWENO spatial reconstructions in one dimension can be employed directly in two or higher dimensions, and this leads to an easier multidimensional simulation. On the other hand, the dimension-bydimension procedure can not be extended to unstructured meshes.

\section{References}

[1] P. Arminjon, D. Stanescu, M.-C. Viallon, A two-dimensional finite volume extension of the LaxFriedrichs and Nessyahu-Tadmor schemes for compressible flow, in Proceedings of the 6th International Symposium on Computational Fluid Dynamics, Vol. IV, Lake Tahoe, NV, 1995, M. Hafez and K. Oshima (Ed.), pp. 7-14.

[2] D. S. Balsara, C. Altmann, C.-D. Munz, M. Dumbser, A sub-cell based indicator for troubled zones in RKDG schemes and a novel class of hybrid RKDG+HWENO shemes, Journal of Computational Physics 226 (2007) 586-620.

[3] D.S. Balsara, C.-W. Shu, Monotonicity preserving weighted essentially non-oscillatory schemes with increasingly high order of accuracy, Journal of Computational Physics 160 (2000) 405-452.

[4] F. Bianco, G. Puppo, G. Russo, High-order central schemes for hyperbolic systems of conservation laws, SIAM Journal on Scientific Computing 21 (1999) 294-322. 
[5] G. Chavent, G. Salzano, A finite-element method for the 1-D water flooding problem with gravity, Journal of Computational Physics 45 (1982) 307-344.

[6] B. Cockburn, C.-W. Shu, The Runge-Kutta local projection $P^{1}$-discontinuous-Galerkin finite element method for scalar conservation law, RAIRO-Modélisation mathématique et analyse numérique 25 (1991) 337-361.

[7] B. Cockburn, C.-W. Shu, TVB Runge-Kutta local projection discontinuous Galerkin finite element method for conservation laws. II. General framework, Mathematics of Computation, 52 (1989) 411-435.

[8] M. Dumbser, D.S. Balsara, E.F. Toro, C.-D. Munz, A unified framework for the construction of one-step finite volume and discontinuous Galerkin schemes on unstructured meshes, Journal of Computational Physics, 227 (2008) 8209-8253.

[9] O. Friedrichs, Weighted essentially non-oscillatory schemes for the interpolation of mean values on unstructured grids, Journal of Computational Physics 144 (1998) 194-212.

[10] D. Gottlieb, L. Lustman, S.A. Orszag, Spectral calculations of one dimensional inviscid flows with shocks, SIAM Journal on Scientific and Statistical Computing 2 (1981) 296-310.

[11] A. Harten, B. Engquist, S. Osher, S. Chakravathy, Uniformly high order accurate essentially nonoscillatory schemes, III, Journal of Computational Physics 71 (1987) 231-303.

[12] C. Hu, C.-W. Shu, Weighted essentially non-oscillatory schemes on triangular meshes, Journal of Computational Physics 150 (1999) 97-127.

[13] T.J. Hughes, A. Brooks, A multidimensional upwind scheme with no crosswind diffusion, in AMD, v.34, Finite Element Methods for Convection Dominated Flows (T.J.Hughes, ed.), ASME, New York, 1979.

[14] T.J. Hughes, A. Brooks, A theoretical framework for Petrov-Galerkin methods with discontinuous weighting functions: Application to the streamline-upwind procedure, Finite Elements in Fluids, Vol. 4 (R.H. Gallagher, ed.), Wiley, New York, 1982.

[15] G. Jiang, C.-W. Shu, Efficient implementation of weighted ENO schemes, Journal of Computational Physics 126 (1996) 202-228.

[16] G. Jiang, E. Tadmor, Nonoscillatory central schemes for multidimensional hyperbolic conservation laws, SIAM Journal on Scientific Computing 19 (1998) 1892-1917.

[17] A. Kurganov, D. Levy, A third-order semidiscrete central scheme for conservation laws and convectiondiffusion equations, SIAM Journal on Scientific Computing 22 (2000) 1461-1488.

[18] P.D. Lax, B. Wendroff, Systems of conservation laws, Communications on Pure and Applied Mathematics 13 (1960) 217-237.

[19] D. Levy, G. Puppo, G. Russo, Central WENO schemes for hyperbolic systems of conservation laws, Mathematical Modelling and Numerical Analysis 33 (1999) 547-571.

[20] D. Levy, G. Puppo, G. Russo, A fourth-order central WENO scheme for multidimensional hyperbolic systems of conservation laws, SIAM Journal on Scientific Computing 24 (2002) 480-506. 
[21] X. Liu, S. Osher, T. Chan, Weighted essentially non-oscillatory schemes, Journal of Computational Physics 115 (1994) 200-212.

[22] X. Liu, E. Tadmor, Third order nonoscillatory central scheme for hyperbolic conservation laws, Numerische Mathematik 79 (1998) 397-425.

[23] Y. Liu, Central schemes on overlapping cells, Journal of Computational Physics 209 (2005) 82-104.

[24] H. Luo, Y. Xia, S. Li, R. Nourgaliev, C. Cai, A Hermite WENO reconstruction-based discontinuous Galerkin method for the Euler equations on tetrahedral grids, Journal of Computational Physics, 231 (2012) 5489-5503.

[25] H. Nessyahu, E. Tadmor, Non-oscillatory central differencing for hyperbolic conservation laws, Journal of Computational Physics 87 (1990) 408-463.

[26] J. Qiu, C.-W. Shu, On the construction, comparision, local characteristic decomposition for high order central WENO schemes, Journal of Computational Physics 183 (2002) 187-209.

[27] J. Qiu, C.-W. Shu, Finite difference WENO schemes with Lax-Wendroff type time discretizations, SIAM Journal on Scientific Computing 24 (2003) 2185-2198.

[28] J. Qiu, C.-W. Shu, Hermite WENO schemes and their application as limiters for Runge-Kutta discontinuous Galerkin method: one dimensional case, Journal of Computational Physics 193 (2004) 115-135.

[29] J. Qiu, C.-W. Shu, Hermite WENO schemes and their application as limiters for Runge-Kutta discontinuous Galerkin method II: Two dimensional case, Computers \& Fluids 34 (2005) 642-663.

[30] J. Shi, C. Hu, C.-W. Shu, A technique of treating negative weights in WENO schemes, Journal of Computational Physics 175 (2002) 108-127.

[31] C.-W. Shu, High order weighted essentially non-oscillatory schemes for convection dominated problems, SIAM Review 51 (2009) 82-126.

[32] C.-W. Shu, S. Osher, Efficient implementation of essentially non-oscillatory shock capturing schemes II, Journal of Computational Physics 83 (1989) 32-78.

[33] E. Tadmor, Convergence of spectral methods for nonlinear conservation laws, SIAM Journal on Numerical Analysis 26 (1989) 30-44.

[34] Z. Tao, F. Li, J. Qiu, High-order central Hermite WENO schemes on staggered meshes for hyperbolic conservation laws, Journal of Computational Physics 281 (2015) 148-176.

[35] Z. Tao, Study of High-order central HWENO finite volume methods for hyperbolic conservation laws and Hamilton-Jacobi equations, doctoral thesis (2016).

[36] P. Woodward, P. Colella, The numerical simulation of two-dimensional fluid flow with strong shocks, Journal of Computational Physics 54 (1984) 115-173.

[37] M. Zennaro, Natural continuous extensions of Runge-Kutta methods, Mathematics of Computation 46 (1986) 119-133. 
[38] F. Zheng and J. Qiu, Directly solving the Hamilton-Jacobi equations by Hermite WENO Schemes, Journal of Computational Physics 307 (2016) 423-445.

[39] J. Zhu, J. Qiu, A class of the fourth order finite volume Hermite weighted essentially non-oscillatory schemes, Science in China Series A: Mathematics 51 (2008) 1549-1560. 